\title{
Density of States and Thouless Formula for Random Unitary Band Matrices
}

\author{
Alain Joye \\ Institut Fourier \\ Université de Grenoble 1, BP 74 \\ 38402 Saint-Martin d'Hères Cedex, France
}

\begin{abstract}
We study the density of states measure for some class of random unitary band matrices and prove a Thouless formula relating it to the associated Lyapunov exponent. This class of random matrices appears in the study of the dynamical stability of certain quantum systems and can also be considered as a unitary version of the Anderson model. We further determine the support of the density of states measure and provide a condition ensuring it possesses an analytic density.
\end{abstract}

\section{Introduction}

The stability of quantum dynamical systems generated by time periodic hamiltonians is sometimes characterized by means of the spectral properties of the corresponding unitary evolution operator over a period, also called monodromy operator, see [Be, [Ho1, [Co3]. Unfortunately, even for this relatively simple time-dependence, except for certain specific models, e.g. $\mathrm{Co} 2, \mathrm{DF}, \mathrm{Bo}$, it is rarely the case that one has enough information about the actual monodromy operator so that a complete spectral analysis can be performed. Therefore, one resorts to different approximation techniques in some specific regimes to say something about the spectrum. For example, KAM inspired techniques, see e.g. Be, Co1, DS, ADE, DLSV, GY], or adiabatic related approaches, see e.g. [Ho2, Ho3, Ho4, N1, J, IN2, have been used to tackle this problem.

In case the complexity of the monodromy operator is important enough to forbid of a complete description of it, one may resort to a statistical modelization. It is the case in particular in the study of the quantum dynamics of electrons confined to a ring threaded by a time dependent magnetic flux, see e.g. the paper $[\mathrm{BB}]$ and references therein. A modelization of this dynamics by means of an effective random monodromy operator taking into account the details of the metallic structure of the ring is considered and tested numerically in $[\mathrm{BB}$. We refer the reader to this paper and [BHJ] for a more detailed account of the construction of the monodromy operator.

Motivated by this approach, the spectral analysis of a class of random and deterministic unitary operators, which contains the above monodromy operator, is performed in [BHJ]. The main characteristics of these unitaries is that, when expressed as matrices in some basis, they display a band structure: more precisely they are five-diagonal. While the construction 
of the set of unitaries studied in [BHJ] is patterned after the above mentionned physical model, we believe it may be useful for a wider class of problems. Moreover, in the regime we consider here, this set of unitaries bears strong resemblances with the Jacobi matrices related to self-adjoint discrete one-dimensional Schrödinger operators.

Another motivation in that direction stems from the recent paper [CMV] where certain unitary infinite matrices associated to the construction of orthonormal polynomials on the unit circle are shown to display the same five-diagonal structure as our set of monodromy operators. These matrices are shown in [CMV] to be unitarily equivalent to unitary operators introduced almost ten years ago in [GT] for the study of a related trigonometric moment problem. Moreover, in the latter paper, some effects of randomness in the coefficients of these operators were investigated.

The goal of the present paper is to pursue the analysis of such random unitaries in the setting considered in the paper [BHJ]. The phases of the matrix elements of the fivediagonal operators are random variables and the deterministic modulus depend on one parameter only: if the phases are all set to zero, what we will call the "free case", the unitary operator depends on a "reflexion" coefficient $r \in] 0,1[$, see below. However, while the analysis of [BHJ] focused on spectral issues, i.e. proving singularity of the almost sure spectrum by means of a unitary version of the Ishii-Pastur theorem and the positivity of the Lyapunov exponent obtained via Furstenberg's Theorem, the main object of the present study of the density of states measure and its links with the corresponding Lyapunov exponent.

More precisely, expressing the density of states as the density of eigenvalues of a series of unitary operators restricted to "boxes", we are able to state this relation as what is known as a Thouless formula. This formula allows to compute the Lyapunov exponent by means of the density of states and to recover the a.c. component of the density of states measure by means of a derivative of the Lyapunov exponent. A consequence of our version of Thouless formula is the extension of some results of [BHJ] providing, in particular, an explicit value of the Lyapunov exponent in these cases. We also prove the validity of the Thouless formula for the deterministic free case, by explicit computations of the relevant quantities. Taking advantage of the analogy of our unitary matrices with the one dimensional discrete Schrödinger operator, we characterize the support of the density of states in term of that of the distribution of the i.i.d. random phases. Finally, we provide an effective criterion ensuring analyticity of the integrated density of states in terms of the exponential decay rate of the Fourier coefficients of the the distribution of the phases. This result relies on some kind of propagation estimates for the free evolution.

We note here also that a Thouless formula is proven for the unitary random operator studied by Geronimo and Teplyaev in [GT]. The corresponding random matrix $V_{\omega}$ is defined in the canonical basis of $l^{2}(\mathbb{Z})$ as well but displays a different structure: for all $k \in \mathbb{Z}$, the vector $V_{\omega} \varphi_{k}$ has non zero coefficients along $\varphi_{j}$, for $j=-\infty, \cdots, k+1$ only. Such matrices are also called Hessenberg matrices. However, the operator under consideration here presents different characteristics from the one of [GT], or [CMV], in particular regarding the way randomness appears in the phases of the matrix elements.

The plan of the paper is as follows. Section 2 is devoted to the definition of the model and its basic properties. The density of states is introduced in the next section and Thouless formula is proven in Section 4. The statements about the support of the density of state and ist analyticity properties are made in Section 5, whereas an Appendix contains some technical items. 


\section{The Model}

We present here the unitary matrices we will be concerned with and recall some of its basic properties to be used later.

The unitary operator we consider has the following explicit form in the canonical basis $\left\{\varphi_{k}\right\}_{k \in \mathbb{Z}}$ of $l^{2}(\mathbb{Z})$

$$
\begin{aligned}
U_{\omega} \varphi_{2 k} & =i r t e^{-i \eta_{2 k}^{\omega}} \varphi_{2 k-1}+r^{2} e^{-i \eta_{2 k}^{\omega}} \varphi_{2 k} \\
& +i r t e^{-i \eta_{2 k+1}^{\omega}} \varphi_{2 k+1}-t^{2} e^{-i \eta_{2 k+1}^{\omega}} \varphi_{2 k+2} \\
U_{\omega} \varphi_{2 k+1} & =-t^{2} e^{-i \eta_{2 k}^{\omega}} \varphi_{2 k-1}+i t r e^{-i \eta_{2 k}^{\omega}} \varphi_{2 k} \\
& +r^{2} e^{-i \eta_{2 k+1}^{\omega}} \varphi_{2 k+1}+i r t e^{-i \eta_{2 k+1}^{\omega}} \varphi_{2 k+2},
\end{aligned}
$$

for any $k \in \mathbb{Z}$. According to [BHJ], the random phases $\left\{\eta_{k}^{\omega}\right\}_{k \in \mathbb{Z}}$ are functions of some physically relevant i.i.d. random variables $\left\{\left(\theta_{k}^{\omega}, \alpha_{k}^{\omega}\right)\right\}_{k \in \mathbb{Z}}$ on the torus given by

$$
\eta_{k}^{\omega}=\theta_{k}^{\omega}+\theta_{k-1}^{\omega}+\alpha_{k}^{\omega}-\alpha_{k-1}^{\omega}
$$

for all $k \in \mathbb{Z}$ and the coefficients $r, t \in] 0,1[$ are interpreted as reflexion and transition coefficients linked by $r^{2}+t^{2}$. We will identify the operator and its matrix representation (2.1). Let us recall that these parameters are assumed to be different from their extreme values 0 and 1 , because in case $r=1 \Longleftrightarrow t=0$ the operator $U_{\omega}$ is diagonal and if $r=0 \Longleftrightarrow t=1$, it is unitarily equivalent to the direct sum of two shifts. Let us finally mention that $U_{\omega}$ is constructed in section 2 of [BHJ] as a product of two unitaries given by infinite direct sums of $2 \times 2$ unitary blocks.

\subsection{Ergodic properties}

More precisely, let us introduce a probabilistic space $(\Omega, \mathcal{F}, \mathbb{P})$, where $\Omega$ is identified with $\left\{\mathbb{T}^{\mathbb{Z}}\right\}, \mathbb{T}$ being the torus, and $\mathbb{P}=\otimes_{k \in \mathbb{Z}} \mathbb{P}_{k}$, where $\mathbb{P}_{2 k}=\mathbb{P}_{0}$ and $\mathbb{P}_{2 k+1}=\mathbb{P}_{1}$ for any $k \in \mathbb{Z}$ are probability distributions on $\mathbb{T}$ and $\mathcal{F}$ the $\sigma$-algebra generated by the cylinders. We introduce the set of random vectors on $(\Omega, \mathcal{F}, \mathbb{P})$ given by

$$
\begin{aligned}
& \beta_{k}=\left(\theta_{k}, \alpha_{k}\right): \Omega \rightarrow \mathbb{T}^{2}, \quad k \in \mathbb{Z}, \\
& \theta_{k}^{\omega}=\omega_{2 k}, \quad \alpha_{k}^{\omega}=\omega_{2 k+1} .
\end{aligned}
$$

The random vectors $\left\{\beta_{k}\right\}_{k \in \mathbb{Z}}$ are thus i.i.d on $\mathbb{T}^{2}$.

We denote by $U_{\omega}$ the random unitary operator corresponding to the random infinite matrix (2.1). In analogy with Jacobi matrices describing the discrete Schrödinger equation, we will also denote the vector $\varphi_{k}$ by the site $k, k \in \mathbb{Z}$.

Introducing the shift operator $S$ on $\Omega$ by

$$
S(\omega)_{k}=\omega_{k+2}, k \in \mathbb{Z}
$$

we get an ergodic set $\left\{S^{j}\right\}_{j \in \mathbb{Z}}$ of translations. With the unitary operator $V_{j}$ defined on the canonical basis of $l^{2}(\mathbb{Z})$ by

$$
V_{j} \varphi_{k}=\varphi_{k-2 j}, \forall k \in \mathbb{Z}
$$

we observe that for any $j \in \mathbb{Z}$

$$
U_{S j \omega}=V_{j} U_{\omega} V_{j}^{*}
$$


Therefore, our random operator $U_{\omega}$ is a an ergodic unitary operator. Now, general arguments on the properties of the spectral resolution of ergodic operators $E_{\omega}(\Delta)$, where $\Delta$ is a Borel set of the torus $\mathbb{T}$, ensure that this projector is weakly measurable, as well as $E_{\omega}^{x}(\Delta)=P_{\omega}^{x} E_{\omega}(\Delta)$, where $x=p . p ., \quad a . c$. and s.c., denote the pure point, absolutely continuous and singular continuous components, see [CL], chapter $\mathrm{V}$. The analysis performed in [BHJ] for the case where $\left\{\left(\theta_{k}^{\omega}, \alpha_{k}^{\omega}\right)\right\}_{k \in \mathbb{Z}}$ are uniformly distributed on the torus shows that the a.c. component of the spectrum of $U_{\omega}$ is almost surely empty.

\subsection{Lyapunov Exponent}

Let us proceed by recalling some facts concerning the Lyapunov exponent. It is shown in $[\mathrm{BB}]$ and $[\mathrm{BHJ}]$ that generalized eigenvectors defined by

$$
\begin{aligned}
& U_{\omega} \psi=e^{i \lambda} \psi, \\
& \psi=\sum_{k \in \mathbb{Z}} c_{k} \varphi_{k}, c_{k} \in \mathbb{C}, \lambda \in \mathbb{C}
\end{aligned}
$$

in our unitary setting can be computed by means of $2 \times 2$ transfer matrices due to the structure of the matrix $U_{\omega}$. They are such that for all $k \in \mathbb{Z},([\mathrm{BHJ}])$

$$
\left(\begin{array}{c}
c_{2 k} \\
c_{2 k+1}
\end{array}\right)=T(k)\left(\begin{array}{c}
c_{2 k-2} \\
c_{2 k-1}
\end{array}\right)
$$

where the randomness lies in the phases $\eta_{k}(\lambda) \equiv \eta_{k}^{\omega}(\lambda)$ defined by

$$
\eta_{k}(\lambda)=\eta_{k}+\lambda
$$

and

$$
\begin{aligned}
T(k)_{11} & =-e^{-i \eta_{2 k-1}(\lambda)} \\
T(k)_{12} & =i \frac{r}{t}\left(e^{-i \eta_{2 k-1}(\lambda)}-1\right) \\
T(k)_{21} & =i \frac{r}{t}\left(e^{i\left(\eta_{2 k}(\lambda)-\eta_{2 k-1}(\lambda)\right)}-e^{-i \eta_{2 k-1}(\lambda)}\right) \\
T(k)_{22} & =-\frac{1}{t^{2}} e^{i \eta_{2 k}(\lambda)}+\frac{r^{2}}{t^{2}}\left(e^{i\left(\eta_{2 k}(\lambda)-\eta_{2 k-1}(\lambda)\right)}+1-e^{-i \eta_{2 k-1}(\lambda)}\right) .
\end{aligned}
$$

Note the properties

$$
T(k) \equiv T\left(\eta_{2 k}(\lambda), \eta_{2 k-1}(\lambda)\right)
$$

whereas $\operatorname{det} T(k)=e^{i\left(\eta_{2 k}-\eta_{2 k-1}\right)}$ is independent of $\lambda$.

Therefore, knowing e.g. the coefficients $\left(c_{0}, c_{1}\right)$, we compute for any $k \in \mathbb{N}$,

$$
\begin{aligned}
\left(\begin{array}{c}
c_{2 k} \\
c_{2 k+1}
\end{array}\right) & =T(k) \cdots T(2) T(1)\left(\begin{array}{c}
c_{0} \\
c_{1}
\end{array}\right) \equiv \Phi(k)\left(\begin{array}{c}
c_{0} \\
c_{1}
\end{array}\right) \\
\left(\begin{array}{c}
c_{-2 k} \\
c_{-2 k+1}
\end{array}\right) & =T(-k+1)^{-1} \cdots T(-1)^{-1} T(0)^{-1}\left(\begin{array}{c}
c_{0} \\
c_{1}
\end{array}\right) \equiv \Phi(-k)\left(\begin{array}{c}
c_{0} \\
c_{1}
\end{array}\right) .
\end{aligned}
$$

The dynamical system at hand being ergodic and the determinant of the transfer matrices being of modulus one, we get the existence of a deterministic Lyapunov exponent $\gamma\left(e^{i \lambda}\right)$, for any $\lambda \in \mathbb{C}$, such that

$$
\lim _{k \rightarrow \pm \infty} \frac{1}{|k|} \ln \|\Phi(k)\|=\gamma\left(e^{i \lambda}\right) \quad \text { a.s.. }
$$

Writing $e^{i \lambda}=z \in \mathbb{C} \backslash\{0\}$, we also know from classical arguments, see e.g. [CFKS, that $\gamma$ is a subharmonic function of $z$. 


\section{Density of States}

Following the standard approach in the self-adjoint case, we start by a definition of the density of states by averaging over the phases and invoking the Riesz-Markov theorem. Then we relate the density of state with alternative definitions in terms of the density of eigenvalues of truncations of the original operator to $l^{2}([M, N])$, as $N-M \rightarrow \infty$.

Definition: The density of states is the (non-random) measure $d k$ on $\mathbb{T}$ defined by

$$
\int_{\mathbb{T}} f\left(e^{i \lambda}\right) d k(\lambda):=\mathbb{E}\left[\left\langle\varphi_{0} \mid f\left(U_{\omega}\right) \varphi_{0}\right\rangle+\left\langle\varphi_{1} \mid f\left(U_{\omega}\right) \varphi_{1}\right\rangle\right] / 2
$$

for any continuous function $f: S^{1} \rightarrow \mathbb{C}$.

The average over the $\varphi_{0}$ and $\varphi_{1}$ matrix elements is motivated by the forms of the matrix (2.1) and shift (2.4). Note also that this definition makes $d k$ a probability measure.

Now we turn to the definition of appropriate finite size unitary matrices constructed from (2.1). There are several possible constructions suited to our purpose. Those we use below result from considering $U_{\omega}$ provided with boundary conditions at certain sites forbidding transitions through these sites. Although such an interpretation is not needed in the sequel, let us mention it can be seen in [BHJ]. There, a more general unitary matrix than (2.1) is considered, whose reflection and transition coefficients $\left(r_{k}, t_{k}\right)$ may depend on the index $k$, whereas (2.1) is a special case with $r_{k}=r$ and $t_{k}=t$. Imposing $t_{N}=0$ there, one gets that the matrix takes a block structure which decouples the sites with indices smaller than $N$ from those with indices larger than $N$.

Let us drop temporarily the sub- and super-scripts $\omega$ in the notation. Fix $N \in \mathbb{Z}$ and consider the unitary operator $U^{2 N}$ on $l^{2}(\mathbb{Z})$ obtained from the original operator $U$ by imposing the following boundary conditions at the sites $2 N$. Let $U^{2 N}$ be defined by (2.1) for $k \notin\{2 N, 2 N+1\}$ where

$$
\eta_{2 N-1}=\eta_{2 N}=\eta_{2 N+1}=\eta_{2 N+2}=0
$$

and, for $k \in\{2 N, 2 N+1\}$

$$
\begin{aligned}
& U^{2 N} \varphi_{2 N}=i t \varphi_{2 N-1}+r \varphi_{2 N} \\
& U^{2 N} \varphi_{2 N+1}=r \varphi_{2 N+1}+i t \varphi_{2 N+2} .
\end{aligned}
$$

Similarly, a boundary condition imposed at site $2 N+1$ defines $U^{2 N+1}$ by (2.1) for $k \notin$ $\{2 N, 2 N+1,2 N+2,2 N+3\}$ where

$$
\eta_{2 N+1}=\eta_{2 N+2}=0
$$

and, for $k \in\{2 N, 2 N+1,2 N+2,2 N+3\}$

$$
\begin{aligned}
& U^{2 N+1} \varphi_{2 N}=i r t e^{-i \eta_{2 N}} \varphi_{2 N-1}+r^{2} e^{-i \eta_{2 N}} \varphi_{2 N}+i t \varphi_{2 N+1} \\
& U^{2 N+1} \varphi_{2 N+1}=-t^{2} e^{-i \eta_{2 N}} \varphi_{2 N-1}+i r t e^{-i \eta_{2 N}} \varphi_{2 N}+r \varphi_{2 N+1} \\
& U^{2 N+1} \varphi_{2 N+2}=r \varphi_{2 N+2}+i r t e^{-i \eta_{2 N+3}} \varphi_{2 N+3}-t^{2} e^{-i \eta_{2 N+3}} \varphi_{2 N+4} \\
& U^{2 N+1} \varphi_{2 N+3}=+i t \varphi_{2 N+2}+r^{2} e^{-i \eta_{2 N+3}} \varphi_{2 N+3}+i r t e^{-i \eta_{2 N+3}} \varphi_{2 N+4} .
\end{aligned}
$$

For any $M \in \mathbb{Z}$, the corresponding operator $U^{M}$ has a the block structure mentionned above and it is unitary. Then, given $(M, N) \in \mathbb{Z}^{2}$ such that $M+4<N$, one defines a unitary matrix $U^{M, N}$ on $l^{2}(\mathbb{Z})$ by imposing boundary conditions at sites $M$ and $N$. By 
construction, $U^{M, N}$ contains an isolated $(N-M) \times(N-M)$ unitary block on $l^{2}([M+1, N])$ we denote by $V^{M, N}$. Introducing the characteristic function $\chi_{M, N}$ of the set $[M+1, N] \in \mathbb{Z}$, we denote by the same symbol the projector on the sites $[M+1, N]$, corresponding to the multiplication operator by $\chi_{M, N}$. Therefore

$$
V^{M, N}=\chi_{M, N} U^{M, N}=U^{M, N} \chi_{M, N}=\chi_{M, N} U^{M, N} \chi_{M, N} .
$$

We now consider two measures related to finite matrices as follows.

Definitions: The measures $d k_{M, N}$ and $\tilde{d k}_{M, N}$ on $\mathbb{T}$ are defined by

$$
\begin{aligned}
& \int_{\mathbb{T}} f\left(e^{i \lambda}\right) d k_{M, N}(\lambda):=\operatorname{tr}\left(f\left(V^{M, N}\right)\right) /(N-M) \\
& \int_{\mathbb{T}} f\left(e^{i \lambda}\right) \tilde{d k_{M, N}}(\lambda):=\operatorname{tr}\left(\chi_{M, N} f(U) \chi_{M, N}\right) /(N-M),
\end{aligned}
$$

for any continuous function $f: S^{1} \rightarrow \mathbb{C}$.

Note that $d k_{M, N}$ is nothing but the counting measure on $\mathbb{T}$ associated with the spectrum of the finite block $V^{M, N}$, and $\tilde{d k}_{M, N}$ that associated with the projection of $U$ on $[M+1, N]$. This former operator is unitary whereas the latter is not.

We denote the trace norm by $\|\cdot\|_{1}$ and first show a slight generalization of [GT] allowing to get

Lemma 3.1 With the above notations, assume

$$
\left\|\left(U^{M, N}-U\right) \chi_{M, N}\right\|_{1}=o(N-N), \text { as } N-M \rightarrow \infty,
$$

then

$$
\lim _{N-M \rightarrow \infty} \frac{1}{N-M}\left(\operatorname{tr}\left(f\left(V^{M, N}\right)\right)-\operatorname{tr}\left(\chi_{M, N} f(U) \chi_{M, N}\right)\right)=0 .
$$

\section{Remark:}

The hypothesis is satisfied in particular if $\operatorname{Rank}\left(U^{M, N}-U\right)<\infty$ and uniformly bounded in $(N, M)$, as is the case with the definitions of $U^{M, N}$ above by means of (3.3, 3.5)

\section{Proof:}

We first note that it is enough to consider functions which are polynomials in $z$ and $\bar{z}, z \in$ $S^{1}$. Any $f \in C\left(S^{1}\right)$ can be approximated by trigonometric polynomials $P_{R}=\sum_{j=-R}^{R} g_{j} e^{i j}$. in such a way that if $\epsilon>0$ is given, there exists $R(\epsilon)<\infty$ so that

$$
\sup _{\theta \in \mathbb{T}}\left|f(\theta)-P_{R(\epsilon)}(\theta)\right| \leq \epsilon .
$$

Hence we get using (3.6),

$$
\begin{aligned}
& \operatorname{tr}\left(f\left(V^{M, N}\right)-\chi_{M, N} f(U) \chi_{M, N}\right)=\operatorname{tr}\left(\chi_{M, N}\left(f\left(U^{M, N}\right)-f(U)\right) \chi_{M, N}\right)= \\
& \operatorname{tr}\left(\chi_{M, N}\left(P_{R(\epsilon)}\left(U^{M, N}\right)-P_{R(\epsilon)}(U)\right) \chi_{M, N}\right)+ \\
& \operatorname{tr}\left(\chi_{M, N}\left(\left(f-P_{R(\epsilon)}\right)\left(U^{M, N}\right)-\left(f-P_{R(\epsilon)}\right)(U)\right) \chi_{M, N}\right),
\end{aligned}
$$

where the trace norm of the last term is bounded by $2 \epsilon(N-M)$, so that it becomes negligeable when divided by $(N-M)$. We are thus to consider $z^{s}$ and $\bar{z}^{s}$, with $s \in \mathbb{N}$. We can write for any $s \geq 1$

$$
U^{s}-\left(U^{N, M}\right)^{s}=\sum_{j=0}^{s-1} U^{j}\left(U-U^{N, M}\right)\left(U^{N, M}\right)^{s-j-1},
$$


so that

$$
\chi_{M, N}\left(U^{s}-\left(U^{N, M}\right)^{s}\right) \chi_{M, N}=\sum_{j=0}^{s-1} \chi_{M, N} U^{j}\left(U-U^{N, M}\right) \chi_{M, N}\left(U^{N, M}\right)^{s-j-1} .
$$

Therefore,

$$
\frac{\operatorname{tr}\left(\chi_{M, N}\left(U^{s}-\left(U^{N, M}\right)^{s}\right) \chi_{M, N}\right)}{N-M} \leq \frac{s\left\|\left(U-U^{N, M}\right) \chi^{M, N}\right\|_{1}}{N-M} .
$$

The same result is true if $s<0$, with all unitaries replaced by their adjoints. Thus, $-R(\epsilon) \leq s \leq R(\epsilon)$ and the hypothesis on the trace norm of $\left(U-U^{N, M}\right) \chi^{M, N}$ yield the result.

Then, restoring the dependence on $\omega$ in the notation, we get by the same arguments as in the self adjoint case, that the density of states is almost surely the limit in the vague sense of the measures $d k_{M, N}$ and $\tilde{d k}_{M, N}$ as $N-M \rightarrow \infty$. A proof is provided in Appendix for completeness.

Proposition 3.1 For any continuous function $f: S^{1} \rightarrow \mathbb{C}$,

$$
\lim _{N-M \rightarrow \infty} \int_{\mathbb{T}} f\left(e^{i \lambda}\right) \tilde{d k}_{M, N}^{\omega}(\lambda)=\int_{\mathbb{T}} f\left(e^{i \lambda}\right) d k(\lambda) \quad \text { a.s. },
$$

and the support of the density of states dk coincides with $\Sigma$, the a.s. spectrum of $U_{\omega}$.

\section{Thouless Formula}

The link between the density of states and the Lyapunov exponent is provided by an analysis of the spectrum of the finite unitary matrices $V^{M, N}$. It reads

Theorem 4.1 [Thouless Formula] For any $z \in \mathbb{C} \backslash\{0\}$

$$
\gamma(z)=2 \int_{\mathbb{T}} \ln \left|z-e^{i \lambda^{\prime}}\right| d k\left(\lambda^{\prime}\right)+\ln \left(1 / t^{2}\right)-\ln |z| .
$$

\section{Remarks:}

0) The identity $\gamma(1 / \bar{z})=\gamma(z)$ holds.

i) It follows from the above formula, as in Theorem 4.6 in [GT], that the integrated density of states is continuous and satisfies

$$
\left|N\left(\lambda_{1}\right)-N\left(\lambda_{2}\right)\right| \leq \frac{\ln \left(2 / t^{2}\right)}{|\ln | e^{i \lambda_{1}}-e^{i \lambda_{2}}||}, \text { where } N(\lambda)=\int_{-\pi}^{\lambda} d k\left(\lambda^{\prime}\right),
$$

by an argument of Craig and Simon [CS.

ii) In case $z=e^{i \lambda} \in S^{1}$, the formula can be cast into the form

$$
\gamma\left(e^{i \lambda}\right)=\int_{\mathbb{T}} \ln \left(\sin ^{2}\left(\left(\lambda-\lambda^{\prime}\right) / 2\right)\right) d k\left(\lambda^{\prime}\right)+\ln \left(4 / t^{2}\right),
$$

from which we recover the estimate $0 \leq \gamma\left(e^{i \lambda}\right) \leq \ln \left(4 / t^{2}\right)$ that follows from the form of the transfer matrices (2.10).

The proof of this version of Thouless formula is given at the end of the section and we proceed with a Corollary and an application of this formula. The Corollary essentially expresses the radial derivative of the Lyapunov exponent as the Poisson integral of the density of states measure $d k$, which allows to recover the a.c. component of $d k$ by a limiting procedure. 
Corollary 4.1 For any $\epsilon>0$ and any $\lambda^{\prime} \in \mathbb{T}$,

$$
\begin{aligned}
& \lim _{\epsilon \rightarrow 0^{+}} \gamma\left(e^{i \lambda^{\prime}} e^{-\epsilon}\right)=\gamma\left(e^{i \lambda^{\prime}}\right), \\
& \frac{\partial}{\partial \epsilon} \gamma\left(e^{i \lambda^{\prime}} e^{ \pm \epsilon}\right)=\mp \int_{T} \frac{1-\left|e^{i \lambda^{\prime}} e^{ \pm \epsilon}\right|^{2}}{\left|e^{i \lambda}-e^{i \lambda^{\prime}} e^{ \pm \epsilon}\right|^{2}} d k(\lambda) \equiv \mp P[d k]\left(e^{i \lambda^{\prime}} e^{ \pm \epsilon}\right) .
\end{aligned}
$$

Therefore, if $n(\lambda) d \lambda / 2 \pi$ denotes the a.c. component of $d k(\lambda)$,

$$
\lim _{\epsilon \rightarrow 0^{+}} \frac{\partial}{\partial \epsilon} \gamma\left(e^{i \lambda^{\prime}} e^{-\epsilon}\right)=n\left(\lambda^{\prime}\right)=\frac{\partial}{\partial \epsilon} \gamma\left(e^{i \lambda^{\prime}}\right)
$$

where the limit and the derivative exist for Lebesgue almost all $\lambda^{\prime} \in \mathbb{T}$.

\section{Remark:}

As in $\left[\mathbf{C S}\right.$, it follows also from the subharmonicity of $\gamma(z)$, that if $\gamma\left(e^{i \lambda_{0}}\right)=0$, then $\gamma: S^{1} \rightarrow \mathbb{R}^{+}$is continuous at $e^{i \lambda_{0}}$.

\section{Proof:}

Let us first consider the second statement with lower indices only. We compute

$$
\gamma\left(e^{i \lambda^{\prime}} e^{-\epsilon}\right)=\epsilon+\ln \left(1 / t^{2}\right)+\int_{\mathbb{T}} \ln \left(1+e^{-2 \epsilon}-e^{-\epsilon} 2 \cos \left(\lambda-\lambda^{\prime}\right)\right) d k(\lambda),
$$

which we can differentiate under the integral sign as long as $\epsilon>0$ to get

$$
\begin{aligned}
\frac{\partial}{\partial \epsilon} \gamma\left(e^{i \lambda^{\prime}} e^{-\epsilon}\right) & =1+\int_{\mathbb{T}} \frac{-2 e^{-2 \epsilon}+e^{-\epsilon} 2 \cos \left(\lambda-\lambda^{\prime}\right)}{1+e^{-2 \epsilon}-e^{-\epsilon} 2 \cos \left(\lambda-\lambda^{\prime}\right)} d k(\lambda) \\
& =\int_{\mathbb{T}} \frac{1-e^{-2 \epsilon}}{1+e^{-2 \epsilon}-e^{-\epsilon} 2 \cos \left(\lambda-\lambda^{\prime}\right)} d k(\lambda)=P[d k]\left(e^{i \lambda^{\prime}} e^{-\epsilon}\right) .
\end{aligned}
$$

The existence for almost all $\lambda^{\prime} \in \mathbb{T}$ of the limit and the first equality in (4.6) is a direct consequence of the above equality. The existence and equality with the derivative at zero for such $\lambda^{\prime}$ follows from the mean value Theorem. To get the first statement, notice that $1+e^{-2 \epsilon}-e^{-\epsilon} 2 \cos (x)>2 e^{-\epsilon}(1-\cos (x))$ in formula (4.7) above yields

$$
\begin{aligned}
& 0 \leq-\ln \left(\left(1+e^{-2 \epsilon}-e^{-\epsilon} 2 \cos \left(\lambda-\lambda^{\prime}\right)\right) / 4\right)<-\ln \left(2 e^{-\epsilon}\left(1-\cos \left(\lambda-\lambda^{\prime}\right)\right) / 4\right)= \\
& \epsilon-\ln \left(\left(1-\cos \left(\lambda-\lambda^{\prime}\right)\right) / 2\right),
\end{aligned}
$$

where the last function is in $L^{1}(\mathbb{T}, d k)$ by Thouless formula. Therefore, an application of the dominated convergence Theorem shows we can take the limit $\epsilon \rightarrow 0$ inside the integral to get the result.

We consider now the properties of $U_{\omega}$ characterized by i.i.d. phases $\theta_{k}^{\omega}$ and $\alpha_{k}^{\omega}$ in the definition (2.2), assuming one set of phases is uniformly distributed on $\mathbb{T}$. In that situation, not only can we can prove the transfer matrices have a (positive) Lyapunov behaviour, but we can also exactly compute the Lyapunov exponent $\gamma\left(e^{i \lambda}\right)$. This shows that in this situation, the spectrum of $U_{\omega}$ is almost surely singular, in view of the unitary version of the Ishii-Pastur Theorem proven in [BHJ]. This strengthens the corresponding results of BHJ], Theorem 4.1 and Propositions 5.4. There Furstenberg's Theorem is applied to prove positivity of the Lyapunov exponent, so that no value for $\gamma\left(e^{i \lambda}\right)$ is provided. 
Theorem 4.2 Let $\left(\theta_{k}^{\omega}\right)_{k \in \mathbb{Z}}$ and $\left(\alpha_{k}^{\omega}\right)_{k \in \mathbb{Z}}$ be i.i.d. on $\mathbb{T}$ and assume the distribution of either the $\theta_{k}^{\omega}$ 's or the $\alpha_{k}^{\omega}$ 's is uniform on $\mathbb{T}$. Then, for any $\lambda \in \mathbb{T}$,

$$
d k(\lambda)=d \lambda / 2 \pi, \text { and } \gamma\left(e^{i \lambda}\right)=\ln \left(1 / t^{2}\right)>0,
$$

therefore,

$$
\sigma\left(U_{\omega}\right)_{a . c}=\emptyset \quad \text { and } \quad \sigma\left(U_{\omega}\right)_{\text {sing. }}=S^{1} \quad \text { almost surely }
$$

\section{Remark:}

The assumption on the distribution of the phases actually implies that the $\eta_{k}$ 's are i.i.d. and uniform on $T$, see Lemma 4.1 below. This explains why the a.s. spectrum coincides with $S^{1}$ and why the density of states is flat.

\section{Proof of Theorem 4.2;}

We first use the following lemma of purely probabilistic nature proven in Appendix.

Lemma 4.1 Under the hypotheses of Theorem 4.2, the $\eta_{k}^{\omega}$ 's are i.i.d. and uniform on T.

Then we show the density of states is uniform for uniformly distributed phases. Expanding $(2.2)$ of the $\eta_{k}(\omega)$ 's we can write for any $n \neq 0$,

$$
\begin{aligned}
& \left\langle\varphi_{j} \mid U_{\omega}^{n} \varphi_{j}\right\rangle=\sum_{\vec{k}=k_{1}, k_{2}, \cdots, k_{n-1}}\left(U_{\omega}\right)_{j, k_{1}}\left(U_{\omega}\right)_{k_{1}, k_{2}} \cdots\left(U_{\omega}\right)_{k_{n-1}, j}= \\
& \sum_{\vec{k}} \exp \left(-i \sum_{l \in \mathcal{L}} p_{l} \eta_{l}(\omega)\right)\left(U_{0}\right)_{j, k_{1}}\left(U_{0}\right)_{k_{1}, k_{2}} \cdots\left(U_{0}\right)_{k_{n-1}, j},
\end{aligned}
$$

where $U_{0}$ corresponds to $U_{\omega}$ when all phases $\eta_{k}=0$ and where $\mathcal{L}$ is a finite set of indices depending on $j, \vec{k}, n$ and $p_{l}$ are integers. Observing that the variables $\eta_{k}(\omega)$ 's all appear with the same sign in (2.1), no compensation can take place between contributions of different matrix elements above and one at least among the integers $p_{l}$, for $l \in \mathcal{L}$ is stricly positive when $n \neq 0$. Using independence and the characterization $\mathbb{E}\left(e^{-i m \eta_{k}(\omega)}\right)=\delta_{m, 0}$ of the uniform distribution, we get

$$
\mathbb{E}\left(\left\langle\varphi_{j} \mid U_{\omega}^{n} \varphi_{j}\right\rangle\right)=\delta_{n, 0} \Longrightarrow \int_{\mathbb{T}} e^{i n \lambda} d k(\lambda)=\delta_{n, 0}
$$

and the first statement follows. The second equality is a consequence of Thouless formula together with the identity

$$
\int_{0}^{2 \pi} \ln \left|1-e^{i \lambda}\right| d \lambda=0 .
$$

The singular nature of the almost sure spectrum of $U_{\omega}$ comes from the unitary version of Ishii-Pastur Theorem proven as Theorem 5.3 in [BHJ], which is independent of the properties of the common distributions of the $\alpha_{k}$ 's and $\theta_{k}$ 's and only requires ergodicity. Finally, Proposition 3.1 yields the result about the support of the a.s. singular spectrum.

We compute here, for the sake of completeness, the density of states and Lyapunov exponent for the deterministic free operator $U_{0}$ corresponding to $U_{\omega}$ in case $\eta_{k}=0, \forall k \in \mathbb{Z}$. In this case, equation (3.16) of Proposition 3.1 becomes a definition of the free density of states $d k_{0}$, provided the limit exists. That the limit exists, is the content of the next 
Lemma 4.2 The free density of states $d k_{0}$ exists when defined for any $f \in C\left(S^{1}\right)$ by

$$
\int_{T} f\left(e^{i \lambda}\right) d k_{0}(\lambda)=\lim _{N-M \rightarrow \infty} \int_{\mathbb{T}} f\left(e^{i \lambda}\right) d \tilde{k}_{M, N}(\lambda) .
$$

As we know essentially everything about the purely a.c. operator $U_{0}$, we can also use a direct approach to perform these computations. In particular, the integrated density of states of $U_{0}$ can be defined as the distribution function on $\mathbb{T}$ of the band functions yielding the spectrum $\Sigma_{0}$ of $U_{0}$. This direct approach of the density of states coincides with the above definition, see the proofs of Proposition 4.1 and Lemma 4.2 in Appendix. We note here that the spectrum of $U_{0}$ consists in the set

$$
\Sigma_{0}=\left\{e^{ \pm i\left(\arccos \left(r^{2}-t^{2} \cos (y)\right)\right)}, y \in \mathbb{T}\right\}
$$

We get in particular that $\Sigma_{0}$ is the support of the density of states whereas $\Sigma_{0}^{c}$ is that of the Lyapunov exponent:

Proposition 4.1 If $N_{0}, d k_{0}$ and $\gamma_{0}$ denote the integrated density of states, the density of states and Lyapunov exponents of $U_{0}$, respectively. We have for $\left.\left.\lambda \in \mathbb{T} \simeq\right]-\pi, \pi\right]$,

$$
\begin{aligned}
d k_{0}(\lambda) & =\left\{\begin{array}{cc}
\frac{|\sin (\lambda)|}{2 \pi \sqrt{t^{4}-\left(r^{2}-\cos (\lambda)\right)^{2}} d \lambda} & \text { if }|\lambda|<\arccos \left(r^{2}-t^{2}\right) \\
0 & \text { otherwise }
\end{array}\right. \\
N_{0}(\lambda) & =\left\{\begin{array}{cc}
\frac{1}{2 \pi} \arccos \left(\frac{r^{2}-\cos (\lambda)}{t^{2}}\right) & \text { if } \lambda \in\left[-\arccos \left(r^{2}-t^{2}\right), 0\right] \\
1-\frac{1}{2 \pi} \arccos \left(\frac{r^{2}-\cos (\lambda)}{t^{2}}\right) & \text { if } \lambda \in\left[0, \arccos \left(r^{2}-t^{2}\right)\right]
\end{array}\right. \\
\gamma_{0}\left(e^{i \lambda}\right) & =\left\{\begin{array}{cc}
0 & \text { otherwise. } \\
\cosh ^{-1}\left(\frac{r^{2}-\cos (\lambda)}{t^{2}}\right) & \text { if }|\lambda| \leq \arccos \left(r^{2}-t^{2}\right)
\end{array}\right.
\end{aligned}
$$

Finally, Thouless formula [4.1) holds true for these quantities with $z=e^{i \lambda}, \lambda \in \mathbb{T}$.

\section{Remarks:}

Note that the density of $d k_{0}(\lambda)$ diverges as $1 / \sqrt{\left|\lambda-\arccos \left(r^{2}-t^{2}\right)\right|}$ at the band edges and behaves as $1 / 2 \pi t$ as $\lambda \rightarrow 0$.

The integrated density of states $N_{0}(\lambda)$ tends to its values 0 and 1 as $\sqrt{\left|\lambda-\arccos \left(r^{2}-t^{2}\right)\right|}$ at the band edges.

Also, in keeping with the fact that $U_{0}$ becomes a shift if $t=1$ and the identity as $r=1$, $N_{0}(\lambda)$ becomes linear in $\lambda$ as $t \rightarrow 1$ and a step function as $r \rightarrow 1$.

The Lyapunov exponent, where non zero, is equivalently given by

$$
\gamma_{0}\left(e^{i \lambda}\right)=\ln \left(\frac{r^{2}-\cos (\lambda)}{t^{2}}+\sqrt{\left(\frac{r^{2}-\cos (\lambda)}{t^{2}}\right)^{2}-1}\right)
$$

It is an even $C^{\infty}$ function of $\lambda$ on $\left\{|\lambda|>\arccos \left(r^{2}-t^{2}\right)\right\}$, strictly increasing on $\left[\arccos \left(r^{2}-\right.\right.$ $\left.\left.t^{2}\right), \pi\right]$. And $d \gamma_{0}\left(e^{i \lambda}\right) / d \lambda$ behaves as $1 / \sqrt{\lambda-\arccos \left(r^{2}-t^{2}\right)}$ as $\lambda \rightarrow \arccos \left(r^{2}-t^{2}\right)^{+}$.

Given Lemma 4.2 above, it is clear that Thouless formula holds for the above quantities. A direct proof of this fact is nevertheless given in Appendix. 


\subsection{Proof of Thouless Formula}

We now turn to the proof of Theorem 4.1. Writing down explicitely the effect of the boundary conditions at $N>M$ on the coefficients of the eigenvector (2.7) we obtain the following relations, which depend on the parity of $N$ and $M$. Let $\psi^{M, N}=\chi_{M, N} \psi$ and consider

$$
V^{M, N} \psi^{M, N}=e^{i \lambda} \psi^{M, N} \quad \text { in } l^{2}[M+1, N] .
$$

We get by inspection,

Lemma 4.3 Assume 4.21) is satisfied. Then, if $M$ is even

$$
\left(\begin{array}{c}
c_{M+2} \\
c_{M+3}
\end{array}\right)=c_{M+1} b_{1}\left(e^{i \lambda}\right) \equiv c_{M+1} \frac{1}{t^{2}}\left(\begin{array}{c}
-i t\left(r-e^{-i \lambda}\right) \\
\left(r-e^{i \lambda}\right)+r\left(r-e^{-i \lambda}\right)
\end{array}\right) .
$$

If $M$ is odd,

$$
\left(\begin{array}{c}
c_{M+1} \\
c_{M+2}
\end{array}\right)=c_{M+1} b_{2}\left(e^{i \lambda}\right) \equiv c_{M+1} \frac{1}{i t}\left(\begin{array}{c}
i t \\
e^{i \lambda}-r
\end{array}\right) .
$$

Similarly, if $N$ is even,

$$
\left(\begin{array}{c}
c_{N-2} \\
c_{N-1}
\end{array}\right)=c_{N} b_{3}\left(e^{i \lambda}\right) \equiv c_{N} \frac{1}{t^{2}}\left(\begin{array}{c}
\left(r-e^{i \lambda}\right)+r\left(r-e^{-i \lambda}\right) \\
-i t\left(r-e^{-i \lambda}\right)
\end{array}\right)
$$

If $N$ is odd,

$$
\left(\begin{array}{c}
c_{N-1} \\
c_{N}
\end{array}\right)=c_{N-1} b_{4}\left(e^{i \lambda}\right) \equiv c_{N-1} \frac{1}{i t}\left(\begin{array}{c}
e^{i \lambda}-r \\
i t
\end{array}\right) .
$$

These relations together with the formulas (2.12) allow to describe the spectrum of $V^{M, N}$ in a convenient manner.

Corollary 4.2 Let $M<N$ be fixed and consider non zero vectors $a_{1}, a_{2} \in \mathbb{C}^{2}$ such that $a_{j}\left(e^{i \lambda}\right) \in\left(b_{j+2}\left(e^{i \lambda}\right) \mathbb{C}\right)^{\perp}, j=1,2$. Then, $e^{i \lambda} \in \sigma\left(V^{M, N}\right)$ iff

$$
\begin{aligned}
& \left\langle a_{1}\left(e^{i \lambda}\right) \mid T(N / 2-1) \cdots T(M / 2+2) b_{1}\left(e^{i \lambda}\right)\right\rangle=0, \\
& \left\langle a_{2}\left(e^{i \lambda}\right) \mid T((N+1) / 2-1) \cdots T(M / 2+2) b_{1}\left(e^{i \lambda}\right)\right\rangle=0, \quad M \text { even }, N \text { odd } \\
& \left\langle a_{1}\left(e^{i \lambda}\right) \mid T(N / 2-1) \cdots T((M+1) / 2+1) b_{2}\left(e^{i \lambda}\right)\right\rangle=0, \quad M \text { odd }, N \text { even } \\
& \left\langle a_{2}\left(e^{i \lambda}\right) \mid T((N+1) / 2-1) \cdots T((M+1) / 2+1) b_{2}\left(e^{i \lambda}\right)\right\rangle=0, \quad M, N \text { odd }
\end{aligned}
$$

Remark:

In particular, a possible choice for the $a_{j}{ }^{\prime}$ is

$$
a_{1}\left(e^{i \lambda}\right)=b_{1}\left(e^{-i \lambda}\right), a_{2}\left(e^{i \lambda}\right)=b_{2}\left(e^{-i \lambda}\right) .
$$

Each of the above quantities denotes a matrix element of a product of transfer matrices of the type (2.12), which depend on $e^{i \lambda}$, and will be linked in the limit $N-M \rightarrow \infty$ to the Lyapunov exponent.

Let $e^{i \lambda}=z \in \mathbb{C} \backslash\{0\}$ and $n_{0}, m_{0} \in \mathbb{Z}$. Defining

$$
\Phi^{m_{0}, n_{0}}(z)=T\left(n_{0}-1\right) \cdots T\left(m_{0}+2\right),
$$

one sees that the matrix elements $\left\langle a_{j}(z) \mid \Phi^{m_{0}, n_{0}}(z) b_{k}(z)\right\rangle$ correspond to those in the above corollary for values $N=2 n_{0}, N=2 n_{0}-1, M=2 m_{0}, M=2 m_{0}+1$, depending on the choice of indices $j, k$. 
Lemma 4.4 For any $z \in \mathbb{C} \backslash S^{1}$ and any indices $j, k=1,2$

$$
\begin{aligned}
\lim _{n_{0}-m_{0} \rightarrow \infty} \frac{1}{2\left(n_{0}-m_{0}\right)} \ln & \left|\left\langle a_{j}(z) \mid \Phi^{m_{0}, n_{0}}(z) b_{k}(z)\right\rangle\right|= \\
& \int_{\mathbb{T}} \ln \left|z-e^{i \lambda^{\prime}}\right| d k\left(\lambda^{\prime}\right)+\ln (1 / t)-\ln \left(|z|^{1 / 2}\right),
\end{aligned}
$$

Proof: We note that for any $k \in \mathbb{Z}$, there exist $2 \times 2$ matrices $A(k), B(k), C(k)$ such that (with $z=e^{i \lambda}$ )

$$
T(k)=z A(k)+B(k)+C(k) / z, \text { where } A(k)=\left(\begin{array}{cc}
0 & 0 \\
0 & -\frac{-e^{i \eta_{2 k}}}{t^{2}}
\end{array}\right)
$$

Also, for any $j=1,2$, there exist vectors $b_{j}^{(k)}, a_{j}^{(k)}, k=-1,0,1$ such that

$$
\begin{aligned}
& a_{k}(z)=z a_{k}^{(1)}+a_{k}^{(0)}+a_{k}^{(-1)} / z, \\
& b_{k}(z)=z b_{k}^{(1)}+b_{k}^{(0)}+b_{k}^{(-1)} / z
\end{aligned}
$$

where $b_{2}^{(-1)}=a_{2}^{(1)}=0$ are the only non zero vectors with the choice (4.27). Thus, taking into account the above property ,

$$
P_{j, k}(z)=z^{n_{0}-m_{0}+(1-k)}\left\langle a_{j}(z) \mid \Phi^{m_{0}, n_{0}}(z) b_{k}(z)\right\rangle
$$

is a polynomial in $z$ of degree $2\left(n_{0}-m_{0}\right)+2-(k+j)$. Let $p_{j, k}$ be the coefficient of the highest power of $z$ of $P_{j, k}$. Then, because of corollary 4.2, we can write

$$
P_{j, k}(z)=p_{j, k} \prod_{l=0}^{\operatorname{deg} P_{j, k}}\left(z-e^{i \lambda_{l}}\right)
$$

where $\left\{e^{i \lambda_{l}}\right\}$ is the set of eigenvalues of $V^{M, N}$ and we compute

$$
\begin{aligned}
& \left|p_{j, k}\right|=\left|\left\langle a_{j}^{(2-j)} \mid \prod_{l=m_{0}+2}^{n_{0}-1} A(l) b_{k}^{(1)}\right\rangle\right|= \\
& \frac{K_{0}}{t^{2\left(n_{0}-m_{0}\right)}}\left|\left\langle\left(\begin{array}{c}
-i t \\
r
\end{array}\right) \mid\left(\begin{array}{ll}
0 & 0 \\
0 & 1
\end{array}\right)^{\left(n_{0}-m_{0}\right)-2}\left(\begin{array}{l}
0 \\
1
\end{array}\right)\right\rangle\right|=\frac{K_{1}}{t^{2\left(n_{0}-m_{0}\right)}}
\end{aligned}
$$

where $K_{0}, K_{1}$ are some constants that depends on $j, k$ and $t$. Therefore, for any $z \in \mathbb{C} \backslash S^{1}$,

$$
\lim _{n_{0}-m_{0} \rightarrow \infty} \frac{\ln \left|P_{j, k}(z)\right|}{\left(n_{0}-m_{0}\right)}=\ln \left(1 / t^{2}\right)+\lim _{n_{0}-m_{0} \rightarrow \infty} \sum_{l=0}^{\operatorname{deg} P_{j, k}} \frac{\ln \left|z-e^{i \lambda_{l}}\right|}{\left(n_{0}-m_{0}\right)}
$$

Introducing the continuous function $f_{z}: S^{1} \rightarrow \mathbb{R}$ given by $f_{z}(x)=\ln |z-x|$, the last term can be written

$$
\lim _{n_{0}-m_{0} \rightarrow \infty} \sum_{l=0}^{\operatorname{deg} P_{j, k}} \frac{f_{z}\left(e^{i \lambda_{j}}\right)}{n_{0}-m_{0}}=2 \lim _{M-N \rightarrow \infty} \frac{\operatorname{tr}\left(f_{z}\left(V^{M, N}\right)\right)}{N-M}=2 \int_{\mathbb{T}} f_{z}\left(e^{i \lambda^{\prime}}\right) d k\left(\lambda^{\prime}\right)
$$

by application of Lemma 3.1 and Proposition 3.1. This ends the proof of the lemma.

Then we make use the following easy lemma 
Lemma 4.5 If $\Phi: \mathbb{C}^{2} \rightarrow \mathbb{C}^{2}$ is linear and $a_{j}, b_{j} \in \mathbb{C}^{2}, j=1,2$ are such that $\operatorname{span}\left(a_{1}, a_{2}\right)=$ $\operatorname{span}\left(b_{1}, b_{2}\right)=\mathbb{C}^{2}$, then $\|\Phi\|:=\max _{j, k}\left|\left\langle a_{j} \mid \Phi b_{k}\right\rangle\right|$ is a norm for $\Phi$,

noting that its hypothesis is satisfied by $a_{k}(z), b_{j}(z)$, for all $z \neq-1$, and of the fact that the Lyapunov exponent is defined independently of the norm used in (2.13) to deduce that (4.29) actually equals half the Lyapunov exponent. Finally, the fact that both the Lyapunov exponent and the right hand side of (4.29) are subharmonic and coincide on $\mathbb{C} \backslash S^{1}$ implies the relation (4.1) on $\mathbb{C}$ as well, by classical arguments, see [CS]. This ends the proof of Thouless formula.

\section{Properties of the Density of States}

We mentionned several times the analogy between our unitary operator $U_{\omega}$ and Jacobi matrices corresponding to the self-adjoint case. In this section we slightly drift away from the physical motivations underlying the study of (2.1) and consider more closely the links between these cases. The analogy is made clearer by the following Lemma which will be useful later.

Lemma 5.1 Denoting unitary equivalence by $\simeq$, we have

$$
U_{\omega} \simeq D_{\omega} S_{0}, \text { with } D_{\omega}=\operatorname{diag}\left\{e^{-i \eta_{k}^{\omega}}\right\}
$$

and

$$
S_{0}=\left(\begin{array}{cccccc}
\ddots & r t & -t^{2} & & & \\
& r^{2} & -r t & & & \\
& r t & r^{2} & r t & -t^{2} & \\
& -t^{2} & -t r & r^{2} & -r t & \\
& & & r t & r^{2} & \\
& & & -t^{2} & -t r & \ddots
\end{array}\right) \simeq U_{0}
$$

where the translation along the diagonal is fixed by $\left\langle\varphi_{2 k-2} \mid S_{0} \varphi_{2 k}\right\rangle=-t^{2}, k \in \mathbb{Z}$.

\section{Remarks:}

In some sense, the Lemma says that, up to unitary equivalence, $U_{\omega}$ is a unitary analog of the one dimensional discrete random Schrödinger operator where the a.c. unitary $S_{0}$ plays the role of the discrete laplacian, the pure point diagonal operator $D_{\omega}$ plays the role of the potential on the sites, and the operator sum is replaced by a product.

We also recall that tridiagonal unitary matrices are spectrally uninteresting as they either correspond to a shift of to infinite direct sums of blocks of size one or two, see Lemma 3.1 in $[\mathrm{BHJ}$.

The Lemma also shows that our operator $U_{\omega}$ is essentially a product of an absolutely continuous unitary and a pure point unitary, whereas it was constructed in Section 2 of [BHJ] as a product of two pure point unitaries.

Proof:

Let us define a collection of rank two operators by

$$
P_{j}=\left|\varphi_{j}\right\rangle\left\langle\varphi_{j}|+| \varphi_{j+1}\right\rangle\left\langle\varphi_{j+1}\right|, \quad j \in \mathbb{Z},
$$

and the unitary $V$ by the direct sum

$$
V=\sum_{j \in \mathbb{Z}}^{\oplus} P_{2 j-1}\left(\begin{array}{cc}
i r & t \\
-i t & r
\end{array}\right) P_{2 j-1} .
$$


It is just a matter of computation to check that we can write

$$
U_{\omega}=\left(U_{\omega} U_{0}^{-1}\right) U_{0} \equiv V^{-1} D_{\omega} V U_{0}=V^{-1} D_{\omega}\left(V U_{0} V^{-1}\right) V \equiv V^{-1}\left(D_{\omega} S_{0}\right) V,
$$

with the required properties for $S_{0}$ and $D_{\omega}$.

Now, forgetting that the phases $\eta_{k}^{\omega}$ are in general correlated random variables, see (2.2), if we consider them as i.i.d., but not necessarily uniformly distributed on $\mathbb{T}$, we get some unitary Anderson-like model. This is where we depart from the physical motivation, as it is recalled in Lemma 4.2 in [BHJ] that independence of the $\eta_{k}$ 's is associated with a uniform distribution.

\subsection{Support of the Density of States}

Nevertheless, assuming the random phases $\left\{\eta_{k}^{\omega}\right\}_{k \in \mathbb{Z}}$ are i.i.d. according to the measure $d \mu$ on $\mathbb{T}$, we can characterize the almost sure spectrum of $U_{\omega}$ in term of the support of $\mu$ and of the spectrum $\Sigma_{0}$ of $U_{0}$.

Theorem 5.1 Under the above hypotheses, the almost sure spectrum of $U_{\omega}$ consists in the set

$$
\Sigma:=\exp (i \operatorname{supp} \mu) \Sigma_{0}=\left\{e^{i \alpha} \Sigma_{0} \mid \alpha \in \operatorname{supp} \mu\right\}
$$

\section{Remark:}

In the case where the $\eta_{k}(\omega)$ are i.i.d. and uniform on $\mathbb{T}$, we recover the fact that the almost sure spectrum of $U_{\omega}$ is $S^{1}$.

\section{Proof:}

To show that $\Sigma$ belongs to the almost sure spectrum, we simply construct Weyl sequences corresponding to the corresponding quasi-energies, with probability one. We know from Section 6 of [BHJ] that for any $e^{i \lambda} \in \Sigma_{0}$, there exists a generalized eigenvector $\psi_{\lambda}$ such

$$
\psi_{\lambda}=\sum_{j \in \mathbb{Z}} c_{j}(\lambda) \varphi_{j}, U_{0} \psi_{\lambda}=e^{i \lambda} \psi_{\lambda}, \text { and } 0<K<\left|c_{j}(\lambda)\right|<1 / K, \forall j \in \mathbb{Z},
$$

for some $K>0$. The last property can be checked also by means of the transfer matrices (2.10)

Let $\alpha \in \operatorname{supp} \mu$. Then, for all $\epsilon>0$, there exists a set $I_{\epsilon} \ni \alpha$ such that $\left|I_{\epsilon}\right| \leq \epsilon$, and $\mu\left(I_{\epsilon}\right)>0$. With the notation $\omega(k)=\eta_{k}(\omega), k \in \mathbb{Z}$, we define for all $n \in \mathbb{N}$ and $k \in \mathbb{Z}$,

$$
A_{n}(k)=\left\{\omega(k n) \in I_{\epsilon}, \omega(k n+1) \in I_{\epsilon}, \cdots, \omega(k n+n-1) \in I_{\epsilon}\right\} .
$$

Due to the assumed independence, we have for any $k, \mathbb{P}\left(A_{n}(k)\right)=\mu\left(I_{\epsilon}\right)^{n}>0$ so that for any $n>0$, by Borel-Cantelli, $\mathbb{P}\left(\cup_{k \in \mathbb{Z}} A_{n}(k)\right)=1$.

Let $\Delta_{n}(k)=\{k n, k n+1, \cdots, k n+n-1\}$ denote the set of indices appearing in $A_{n}(k)$ and consider now

$$
\psi_{n, k}(\lambda)=\sum_{j \in \Delta_{n}(k)} c_{j}(\lambda) \varphi_{j}=\chi\left(\Delta_{n}(k)\right) \psi(\lambda),
$$

where $\chi\left(\Delta_{n}(k)\right)$ is the projector on the span of $\left\{\varphi_{j}\right\}_{j \in \Delta_{n}(k)}$ Because of (5.7),

$$
U_{0} \psi_{n, k}(\lambda)=e^{i \lambda} \psi_{n, k}(\lambda)+R_{k n}^{-}(\lambda)+R_{k(n+1)}^{+},
$$


where the vectors $R_{j}^{ \pm}$have at most four components close to the index $j$ and

$$
\left\|R_{j}^{ \pm}\right\| \leq R, \text { where } R \text { is uniform in } j .
$$

Also, by construction of $A_{n}(k), U_{0}$ and $U_{\omega}$, we have

$$
\begin{aligned}
\left\|U_{\omega} \psi_{n, k}(\lambda)-e^{i \alpha} U_{0} \psi_{n, k}(\lambda)\right\| & \leq\left\|\left(U_{\omega}-e^{i \alpha} U_{0}\right) \chi\left(\Delta_{n}(k)\right) \mid\right\| \psi_{n, k}(\lambda) \| \\
& =O(\epsilon)\left\|\psi_{n, k}(\lambda)\right\|,
\end{aligned}
$$

where the estimate $O(\epsilon)$ is uniform in $n$ and $k$. Therefore, for all $\epsilon>0$ and all $n>0$, there exists, with probability one, a $k$ such that $A_{n}(k)$ and the corresponding $\psi_{n, k}(\lambda)$ have the above properties so that

$$
\begin{aligned}
& \left\|U_{\omega} \psi_{n, k}(\lambda)-e^{i(\alpha+\lambda)} \psi_{n, k}(\lambda)\right\| /\left\|\psi_{n, k}(\lambda)\right\|= \\
& \left(\left\|\left(U_{\omega}-e^{i \alpha} U_{0}\right) \psi_{n, k}(\lambda)+e^{i \alpha}\left(U_{0}-e^{i \lambda}\right) \psi_{n, k}(\lambda)\right\|\right) /\left\|\psi_{n, k}(\lambda)\right\| \leq \\
& O(\epsilon)+2 R /\left\|\psi_{n, k}(\lambda)\right\|=O(\epsilon+1 / n) .
\end{aligned}
$$

It remains to chose $n=[1 / \epsilon]$ to conclude that $e^{i(\alpha+\lambda)} \in \sigma\left(U_{\omega}\right)$ almost surely.

Let us now show that $S^{1} \backslash \Sigma$ belongs to the resolvent set of $U_{\omega}$. In order to do so we use Lemma 5.1 Therefore, we can consider as well the spectrum of the product $D_{\omega} S_{0}$ to which the perturbation theory recalled in Chap.1, $\S 11$ of Yaf for example, applies. In particular, dropping the $\omega$ in the notation as randomness plays no role here, if we know that for all $j \in \mathbb{Z}, \eta_{j} \in[\alpha, \beta] \subset \mathbb{T}$, then $\sigma(D) \subseteq\left(\delta_{1}, \delta_{2}\right)$ where $\left(\delta_{1}, \delta_{2}\right)$ denotes the corresponding arc on the unit circle swept in the positive direction from $\delta_{1} \in S^{1}$ to $\delta_{2} \in S^{1}$. We denote by $\left|\left(\delta_{1}, \delta_{2}\right)\right|$ the length on the torus of this arc. Since $\sigma\left(S_{0}\right)=\Sigma_{0}$ corresponds to the symmetric arc $\left(e^{-i \arccos \left(r^{2}-t^{2}\right)}, e^{i \arccos \left(r^{2}-t^{2}\right)}\right)$, perturbation theory tells us that after (multiplicative) perturbation by $S_{0}$, the spectrum of $U \simeq D S_{0}$ is a subset of an arc of wider aperture than $\left(\delta_{1}, \delta_{2}\right)$. Quantitatively, Theorem 8, p.65 in Yaf tells us that the arc $\left(e^{i \arccos \left(r^{2}-t^{2}\right)} \delta_{2}, e^{-i \arccos \left(r^{2}-t^{2}\right)} \delta_{1}\right)$ belongs to the resolvent set of $U$, provided $\left|\left(\delta_{1}, \delta_{2}\right)\right|<\left|\left(e^{i \arccos \left(r^{2}-t^{2}\right)}, e^{-i \arccos \left(r^{2}-t^{2}\right)}\right)\right|$. This condition simply insures that the subset of the resolvent set we are talking about is not reduced to the empty set. This is enough to get the result in case the support of $\mu$ is such that $\Sigma$ is connected. In case this set is not connected, as $\left|\Sigma_{0}\right|>0$, it consists of a finite set of connected components, each of which can be associated with the convex hull of sufficiently far apart subsets of the support of $\mu$. Denoting these subsets by $m_{j}, j=1, \cdots, N$ and the associated arcs on $S^{1}$ by $\left(M_{1}(j), M_{2}(j)\right)$, we have that the spectrum of $D$ is the disjoint union of subsets $\sigma_{j}$ satisfying $\sigma_{j} \subseteq\left(M_{1}(j), M_{2}(j)\right)$. The same argument as above says that the spectrum of $D S_{0}$ is confined to the finite union of $\operatorname{arcs}\left(\left(e^{i \arccos \left(r^{2}-t^{2}\right)} M_{1}(j),\left(e^{-i \arccos \left(r^{2}-t^{2}\right)} M_{2}(j)\right)\right.\right.$, which ends the proof of the Theorem.

\subsection{Analyticity of the density of states}

At the price of some combinatorics, we can further exploit the relation (4.12) in order to obtain a condition on the common distribution of the $\eta_{k}$ 's ensuring the analyticity of the density of states. Recall that a function $f$ on $\mathbb{T}$ is analytic, if and only if its Fourier coefficients $\hat{f}$ satisfy an estimate of the form

$$
|\hat{f}(n)| \leq A e^{-B|n|}, \forall n \in \mathbb{Z},
$$

for some positive constants $A, B$. We have 
Theorem 5.2 Assume the $\eta_{k}$ 's are distributed according to a law that has an analytic density $f$ characterized by the estimate (5.14) with $A, B>0$. Then, if

$$
B>\ln (1+2 r t)+\ln A,
$$

the density of states dk admits an analytic density, so that the integrated density of states $N$ is analytic as well.

\section{Remarks:}

As $\hat{f}(0)=\int_{\mathbb{T}} f(\eta) d \eta=1, A \geq 1$.

When the Theorem applies, it prevents the Lyapunov exponent from being zero on a set of positive measure.

This result has to be compared with the Proposition VI. 3.1. of CL stating a similar result for the $d$-dimensional Anderson model.

As an immediate consequence, using $r^{2}+t^{2}=1$, we get the following

Corollary 5.1 If the $\eta_{k}$ 's have an analytic density $f$, characterized by (5.14) with $B>$ $\ln A$, then there exist $r^{+}(f)$ and $r^{-}(f)$ in $] 0,1[$ such that the density of states is analytic provided the reflexion coefficient $r$ satisfies $1>r>r^{+}(f)$ or $0<r<r^{-}(f)$. If $B>\ln (2 A)$, The density of state is analytic $\forall r \in[0,1]$.

\section{Remark:}

It is easy to check that in both the extreme cases $r=1$ and $r=0$, the density of states is analytic. Indeed, if $r=1, d k(\lambda)=f(\lambda) d \lambda$, where $f$ is the density of the $\eta_{k}$ 's, whereas if $r=0, d k(\lambda)=d \lambda /(2 \pi)$.

\section{Proof of Theorem [5.2;}

By hypothesis, for any $n \in \mathbb{Z}$,

$$
\left|\Phi_{\eta}(n)\right|=\left|\int_{\mathbb{T}} e^{i \eta n} f(\eta) d \eta\right| \leq A e^{-B|n|}
$$

Then, in (4.12) above, $\sum_{l \in \mathcal{L}} p_{l}=n$, so that using independence

$$
\left|\mathbb{E}\left\langle\varphi_{j} \mid U_{\omega}^{n} \varphi_{j}\right\rangle\right| \leq A^{n} e^{-B n} \sum_{k_{1}, k_{2}, \cdots, k_{n-1}}\left|\left(U_{0}\right)_{j, k_{1}}\right|\left|\left(U_{0}\right)_{k_{1}, k_{2}}\right| \cdots\left|\left(U_{0}\right)_{k_{n-1}, j}\right|
$$

Here the sum carries over a set of indices that form paths of length $n+1$ from index $j$ to index $j$. The allowed paths are those giving rise to non zero matrix elements $\left(U_{0}\right)_{l, m}$ in the sum above. In order to compute this last sum, we proceed as follows. Let us introduce more general $j$-dependent subsets $\mathcal{C}_{n-1}(j)$ of indices of $\mathbb{Z}^{n-1}$ that appear in the computation of the matrix element $\left\langle\varphi_{0} \mid U_{\omega}^{n} \varphi_{j}\right\rangle$. This set consists of paths of the form $\left\{k_{0}=0, k_{1}, k_{2}, \cdots, k_{n-1}, k_{n}=j\right\}$ of length $n+1$ in $\mathbb{Z}$ from 0 to $j$ with the condition that

$$
\begin{array}{ll}
k_{m+1}-k_{m} \in\{0,+1,-1,+2\} & \text { if } \quad k_{m} \text { is odd } \\
k_{m+1}-k_{m} \in\{0,+1,-1,-2\} & \text { if } \quad k_{m} \text { is even, }
\end{array}
$$

for all $m=0,1, \cdot, n-1$. Let us define

$$
S_{n-1}(j):=\sum_{\mathcal{C}_{n-1}(0)}\left|\left(U_{0}\right)_{0, k_{1}}\right|\left|\left(U_{0}\right)_{k_{1}, k_{2}}\right| \cdots\left|\left(U_{0}\right)_{k_{n-1}, j}\right|,
$$

where the matrix elements $\left|\left(U_{0}\right)_{l, m}\right|$ are given by $r^{2}, r t$ and $t^{2}$ respectively, when $|l-m|$ equals 0,1 and 2 respectively. This quantity actually gives a crude upper bound on the 
probability to go from site 0 to $j$ in $n$ time steps, under the free evolution. It is crude in the sense that it does not take the phases into account during that free evolution.

We are actually interested in the computation of $S_{n-1}(0)$ and of the similar quantity appearing in the computation of $\left\langle\varphi_{1} \mid U_{\omega}^{n} \varphi_{1}\right\rangle$, which correspond the the sum in the right hand side of (5.17), in the asymptotic regime $n \rightarrow \infty$. The case of the matrix element $\left\langle\varphi_{1} \mid U_{\omega}^{n} \varphi_{1}\right\rangle$ being similar, we only consider $S_{n-1}(0)$.

The plan is to use a transfer matrix formalism to evaluate the generating function associated with $S_{n-1}(j)$ and then to compute the asymptotics of $S_{n-1}(0)$. In view of (5.17), the following proposition implies the Theorem.

Proposition 5.1 For some constant $c>0$,

$$
S_{n-1}(0)=\frac{c(r+t)^{2 n}}{\sqrt{n}}(1+o(1)) \text { as } n \rightarrow \infty .
$$

\section{Proof of Proposition [5.1;}

Let

$$
P_{n}(x)=\sum_{-2 n \leq j \leq 2 n} S_{n-1}(j) x^{j}
$$

be this generating function which we split into two parts $P_{n}(x)=P_{n}^{+}(x)+P_{n}^{-}(x)$ where

$$
P_{n}^{ \pm}(x)=\sum_{\substack{-2 n \leq j \leq 2 n \\ j \text { even } \\ \text { odd }}} S_{n-1}(j) x^{j}
$$

Clearly we have for $n=0,1$,

$$
P_{0}^{+}(x)=r^{2}, P_{0}^{-}(x)=0, P_{1}^{+}(x)=r^{2}+t^{2} x^{-2}, P_{1}^{-}(x)=r t\left(x+x^{-1}\right) .
$$

It is readily shown by induction that a transfer matrix allows to compute $P_{n}(x)$ for any $n$ :

Lemma 5.2 For any $n \geq 0$,

$$
\left(\begin{array}{c}
P_{n+1}^{+}(x) \\
P_{n+1}^{-}(x)
\end{array}\right)=\left(\begin{array}{cc}
r^{2}+t^{2} x^{-2} & r t\left(x+x^{-1}\right) \\
r t\left(x+x^{-1}\right) & r^{2}+t^{2} x^{2}
\end{array}\right)\left(\begin{array}{c}
P_{n}^{+}(x) \\
P_{n}^{-}(x)
\end{array}\right),
$$

with $P_{0}^{+}(x)=r^{2}, P_{0}^{-}(x)=0$.

Denoting by $T(x)$ the transfer matrix defined in this Lemma, and introducing the parameter

$$
\tau=t / r \in] 0, \infty[
$$

we rewrite it as

$$
T(x)=r^{2}\left(\begin{array}{cc}
1+\tau^{2} x^{-2} & \tau\left(x+x^{-1}\right) \\
\tau\left(x+x^{-1}\right) & 1+\tau^{2} x^{2}
\end{array}\right) .
$$

We will consider first the case $t \neq r \Longleftrightarrow \tau \neq 1$. The case $\tau=1$, for which more can be said about $S_{n-1}(j)$, see Proposition [5.2, is dealt with below. 


\subsubsection{Case $\tau \neq 1$}

The eigenvalues of $T(x)$ are given by $r^{2}$ times $\lambda_{ \pm}(x)$, where

$$
\lambda_{ \pm}(x)=\left\{1+\tau\left(x^{2}+x^{-2}\right) / 2 \pm \sqrt{\left(1+\tau\left(x^{2}+x^{-2}\right) / 2\right)^{2}-\left(1-\tau^{2}\right)^{2}}\right\},
$$

so that

$$
T^{n}(x)=r^{2 n} A(x)\left(\begin{array}{cc}
\lambda_{+}^{n}(x) & 0 \\
0 & \lambda_{-}(x)^{n}
\end{array}\right) A(x)^{-1}
$$

with

$$
A(x)=\left(\begin{array}{cc}
\lambda_{+}(x)-\left(1+\tau^{2} x^{2}\right) & \lambda_{-}(x)-\left(1+\tau^{2} x^{2}\right) \\
\tau\left(x+x^{-1}\right) & \tau\left(x+x^{-1}\right)
\end{array}\right)
$$

For the moment, $x$ is just book keeping parameter, so that we ignore the potential problems of the definition of $A(x)$ in case the eigenvalues are degenerate and we further compute

$$
\begin{aligned}
& \left(\begin{array}{c}
P_{n}^{+}(x) \\
P_{n}^{-}(x)
\end{array}\right)=T^{n}(x)\left(\begin{array}{c}
r^{2} \\
0
\end{array}\right)= \\
& \frac{r^{2 n} \tau\left(x+x^{-1}\right)}{2 \sqrt{\left(1+\tau\left(x^{2}+x^{-2}\right) / 2\right)^{2}-\left(1-\tau^{2}\right)^{2}}} \times \\
& \left(\begin{array}{c}
\lambda_{+}(x)^{n+1}-\lambda_{-}(x)^{n+1}-\left(\lambda_{+}(x)^{n}-\lambda_{-}(x)^{n}\right)\left(1+\tau^{2} x^{2}\right) \\
\tau\left(x+x^{-1}\right)\left(\lambda_{+}(x)^{n}-\lambda_{-}(x)^{n}\right)
\end{array}\right) .
\end{aligned}
$$

We note at this point that one checks, using the binomial Theorem, that despite the presence of square roots in the expressions for $P_{n}^{ \pm}(x)$, these quantities actually are given by finite Laurent expansions in $x$, as they should. Focusing on $P_{n}^{+}(x)$ we can rewrite with the shorthand $\sqrt{\cdot}$ for the square root of the denominator above

$$
\begin{aligned}
& P_{n}^{+}(x)= \\
& \quad \frac{r^{2 n} \tau\left(x+x^{-1}\right)}{2 \sqrt{\cdot}}\left(\left(\lambda_{+}(x)^{n}-\lambda_{-}(x)^{n}\right) \frac{\tau^{2}}{2}\left(x^{-2}+x^{2}\right)+\frac{\sqrt{\cdot}}{2}\left(\lambda_{+}(x)^{n}+\lambda_{-}(x)^{n}\right)\right) .
\end{aligned}
$$

The quantity of interest to us is $S_{n-1}(0)$, the coefficient of $x^{0}$ in the expansion of $P_{n}^{+}(x)$. Substituting $e^{i \theta}$ for $x$ in $P_{n}^{+}$, we get a trigonometric polynomial whose zero'th Fourier coefficient is obtained by integration

$$
S_{n-1}(0)=\int_{\mathbb{T}} P_{n}^{+}\left(e^{i \theta}\right) d \theta /(2 \pi)
$$

It remains to perform the asymptotic analysis of the above integral as $n \rightarrow \infty$. It is a matter of routine to verify the following propereties: The eigenvalues, as functions of $\theta \in \mathbb{T} \simeq]-\pi, \pi]$, are continuous. If $\tau<1$, they are real valued, with discontinuity of the derivative at $\theta= \pm \pi / 2$, where they cross and are given by $1-\tau^{2}$. At all other values of $\theta$, they are $C^{\infty}$ and they satisfy

$$
\lambda_{+}\left(e^{i \theta}\right)>\lambda_{-}\left(e^{i \theta}\right), \text { with } \lambda_{+}\left(e^{i \theta}\right)>1-\tau^{2} .
$$

If $\tau>1$, the eigenvalues become complex conjugate. Let $\theta_{c}=\arccos \left(\frac{\tau^{2}-2}{\tau^{2}}\right) / 2$ be the critical value where the square root becomes zero. If $\theta \in\left[\theta_{c}, \pi-\theta_{c}\right] \cup\left[-\pi+\theta_{c},-\theta_{c}\right]$, the eigenvalues are complex conjugate, of modulus $\left|1-\tau^{2}\right|$. Otherwise they are real valued, and satisfy (5.32) as well. Therefore, the asymptotics as $n \rightarrow \infty$ of (5.31) is determined by $\lambda_{+}$only. Moreover, in both cases, $\ln \left(\lambda_{+}\left(e^{i \theta}\right)\right)$ admits non degenerate maxima at $\theta=0$ and $\pi$, where $\lambda_{+}$reaches its maximum value $\left(1+\tau^{2}\right)$. Therefore, Laplace's method yields the asymptotics of the Proposition. 


\subsection{2 case $\tau=1$}

The course of the proof being the same, it is presented in Appendix. However, instead of computing $S_{n-1}(0)$ as $n \rightarrow \infty$, we can get exact forms for all $S_{n-1}(j)$ 's. The Proposition we actually show is

\section{Proposition 5.2}

$$
\begin{aligned}
& S_{n-1}(j)=\frac{1}{2^{n}}\left(\begin{array}{c}
2 n-1 \\
j / 2+n
\end{array}\right), \quad-2 n \leq j \leq 2(n-1), \quad j \text { even } \\
& S_{n-1}(j)=\frac{1}{2^{n}}\left(\begin{array}{c}
2 n-1 \\
(j-1) / 2+n
\end{array}\right), \quad-2 n+1 \leq j \leq 2 n-1, \quad j \text { odd }
\end{aligned}
$$

\section{Remark:}

Of course, Stirling's formula for $n$ large yields proposition [5.1] with $r=t=1 / \sqrt{2}$ :

$$
S_{n-1}(0)=\frac{1}{2^{n}}\left(\begin{array}{c}
2 n-1 \\
n
\end{array}\right) \simeq \frac{2^{n}}{\sqrt{\pi n}}
$$

\section{Appendix}

\section{Proof of Proposition 3.1;}

We have by definition,

$$
\int_{\mathbb{T}} f\left(e^{i \lambda}\right) \tilde{d k}_{M, N}^{\omega}(\lambda)=\frac{1}{N-M} \sum_{j=M+1}^{N}\left\langle\varphi_{j} \mid f\left(U_{\omega}\right) \varphi_{j}\right\rangle
$$

where, depending on the parity of $M$ and $N$ and due to the fact that $f$ is uniformly bounded, the right hand side can be rewritten as

$$
\begin{aligned}
& \frac{1}{N-M}\left(\sum_{k=(M+1) / 2}^{N / 2}\left\langle\varphi_{2 k} \mid f\left(U_{\omega}\right) \varphi_{2 k}\right\rangle+\left\langle\varphi_{2 k+1} \mid f\left(U_{\omega}\right) \varphi_{2 k+1}\right\rangle\right)+O_{f}\left(\frac{1}{N-M}\right)= \\
& \frac{1}{N-M}\left(\sum_{k=(M+1) / 2}^{N / 2}\left\langle\varphi_{0} \mid f\left(U_{S^{k}(\omega)}\right) \varphi_{0}\right\rangle+\left\langle\varphi_{1} \mid f\left(U_{S^{k}(\omega)}\right) \varphi_{1}\right\rangle\right)+O_{f}\left(\frac{1}{N-M}\right) .(6.2)
\end{aligned}
$$

Now, by Birkhoff ergodic theorem, there exists $\Omega_{f}$ of measure one such that for all $\omega \in \Omega_{f}$,

$$
\lim _{N-M \rightarrow \infty} \frac{1}{N-M} \sum_{k=(M+1) / 2}^{N / 2}\left\langle\varphi_{j} \mid f\left(U_{S^{k}(\omega)}\right) \varphi_{j}\right\rangle=\frac{1}{2} \mathbb{E}\left(\left\langle\varphi_{j} \mid f\left(U_{\omega}\right) \varphi_{j}\right\rangle\right), \forall j \in \mathbb{Z},
$$

therefore,

$$
\frac{1}{N-M} \operatorname{tr}\left(\chi^{M, N} f\left(U_{\omega}\right)\right) \rightarrow \frac{1}{2}\left(\mathbb{E}\left(\left\langle\varphi_{0} \mid f\left(U_{\omega}\right) \varphi_{0}\right\rangle+\left\langle\varphi_{0} \mid f\left(U_{\omega}\right) \varphi_{0}\right\rangle\right)\right) .
$$

Then, $C\left(S^{1}\right)$ being separable, we have the existence of a countable set of $\left\{f_{j}\right\}_{j \in \mathbb{N}}$, dense in $C\left(S^{1}\right)$, for which the above is true, on a set of probability one, which proves the almost sure convergence stated in the proposition.

Now assume $e^{i \lambda_{0}} \notin \Sigma$ and take a continuous non negative $f$ such that $f\left(e^{i \lambda_{0}}\right)=1$ and $\left.f\right|_{\Sigma}=0$. Then $f\left(U_{\omega}\right)=0$ a.s. so that $\int f\left(e^{i \lambda}\right) d k(\lambda)=0$ and $e^{i \lambda_{0}} \notin \operatorname{supp} k$. 
Conversely, if $e^{i \lambda_{0}} \notin \operatorname{supp} k$, there exists a non negative continuous $f$ with $f\left(e^{i \lambda_{0}}\right)=1$ and $\int f\left(e^{i \lambda}\right) d k(\lambda)=0$. Hence, a.s., $\left\langle\varphi_{0} \mid f\left(U_{\omega}\right) \varphi_{0}\right\rangle+\left\langle\varphi_{1} \mid f\left(U_{\omega}\right) \varphi_{1}\right\rangle=0$, therefore, by ergodicity, $\left\langle\varphi_{j} \mid f\left(U_{\omega}\right) \varphi_{j}\right\rangle=0$ a.s. for any $j$ and $f\left(U_{\omega}\right)=0$. As $f$ is continuous and equals one at $e^{i \lambda_{0}}$, we get that $e^{i \lambda_{0}} \notin \Sigma$.

\section{Proof of Lemma 4.1 ;}

We only deal with the case where the $\theta_{k}^{\omega}$ 's are i.i.d. and uniform, the other case beeing similar. Let $\Phi_{\eta}(n)=\mathbb{E}\left(e^{i n \eta_{k}^{\omega}}\right)$ be the characteristic function of the random variable $\eta_{k}^{\omega}$, and similarly for $\alpha_{k}^{\omega}$, and $\Phi_{\theta}(n)=\delta_{n, 0}$. Then, using independence,

$$
\Phi_{\eta}(n)=\Phi_{\theta}(n)^{2} \Phi_{\alpha}(n) \Phi_{\alpha}(-n)=\delta_{n, 0}\left|\Phi_{\alpha}(n)\right|^{2}=\delta_{n, 0},
$$

so that the $\eta_{k}$ 's are uniformly distributed. Consider now

$$
\Phi_{\eta_{k_{0}}, \eta_{k_{1}}, \cdots, \eta_{k_{j}}}\left(n_{0}, n_{1}, \cdots, n_{j}\right)=\mathbb{E}\left(e^{i \sum_{l=0}^{j} k_{l} \eta_{l}}\right) .
$$

We can assume the $k_{j}$ 's are ordered and we observe that $\eta_{k}$ and $\eta_{k+j}$ are independent as soon as $j \geq 2$, see (2.2). Therefore, we can consider consecutive indices $k_{l}$ and deal with

$$
\begin{aligned}
& \Phi_{\eta_{k}, \eta_{k+1}, \cdots, \eta_{k+j}}\left(n_{1}, n_{2}, \cdots, n_{j}\right)= \\
& \mathbb{E}\left(e^{\left.i n_{0} \theta_{k-1}+i\left(n_{0}+n_{1}\right) \theta_{k}+\cdots+i\left(n_{j-1}+n_{j}\right) \theta_{k+j-1}+n_{j} \theta_{j}\right)} \mathbb{E}(f(\alpha, \vec{n})),\right.
\end{aligned}
$$

where the second expectation contains $\alpha_{k}$ 's only. Then

$$
\begin{aligned}
& \Phi_{\eta_{k}, \eta_{k+1}, \cdots, \eta_{k+j}}\left(n_{1}, n_{2}, \cdots, n_{j}\right)= \\
& \Phi_{\theta}\left(n_{0}\right) \Phi_{\theta}\left(n_{0}+n_{1}\right) \cdots \Phi_{\theta}\left(n_{j-1}+n_{j}\right) \Phi_{\theta}\left(n_{j}\right) \mathbb{E}(f(\alpha))= \\
& \delta_{n_{0}, 0} \delta_{n_{1}, 0} \cdots \delta_{n_{j}, 0} \mathbb{E}(f(\alpha, \vec{n}))=\delta_{\vec{n}, \overrightarrow{0}} \mathbb{E}(f(\alpha, \overrightarrow{0}))=\delta_{\vec{n}, \overrightarrow{0}},
\end{aligned}
$$

whith the obvious notation, which yields the result.

\section{Proof of Proposition 4.1;}

We first prove this Proposition with the definition of the density of states as the distribution function of the "band functions" of $U_{0}$, to be defined below. Then we'll see in the course of the proof of Lemma 4.2 below the equivalence with the definiton as an average counting measure. The proof of Proposition 6.2 in [BHJ] shows that $U_{0}$ on $l^{2}(\mathbb{Z})$ is unitarily equivalent to the operator multiplication by the matrix

$$
V(x)=\left(\begin{array}{cc}
r^{2}-t^{2} e^{2 i x} & 2 i t r \cos (x) \\
2 i t r \cos (x) & r^{2}-t^{2} e^{-2 i x}
\end{array}\right) \quad \text { on } L^{2}(\mathbb{T}) \simeq L_{+}^{2}(\mathbb{T}) \oplus L_{-}^{2}(\mathbb{T}),
$$

by the unitary mapping that sends $\varphi_{k} \mapsto e^{i k x} / \sqrt{2 \pi}$, and where $L_{ \pm}^{2}(\mathbb{T})$ is the susbspace generated by even/odd harmonics $\left\{e^{i k x}\right\}_{k \in \mathbb{Z}}$. The eigenvalues of $V(x)$ are

$$
\lambda_{ \pm}(x)=e^{ \pm i \alpha(x)}, \text { where } \alpha(x)=\arccos \left(r^{2}-t^{2} \cos (2 x)\right) .
$$

We note that $\lambda_{ \pm}(x)=\lambda_{ \pm}(-x)$ and

$$
V(x)=J V(-x) J \text { where } J=\left(\begin{array}{ll}
0 & 1 \\
1 & 0
\end{array}\right)
$$

Hence, the corresponding eigenvectors $\chi_{ \pm}(x)$ satisfy

$$
V(x) \chi_{ \pm}(x)=\lambda_{ \pm}(x) \chi_{ \pm}(x) \text { and } V(x) J \chi_{ \pm}(-x)=\lambda_{ \pm}(x) J \chi_{ \pm}(-x)
$$


so that $\chi_{ \pm}(x)$ and $J \chi_{ \pm}(-x)$ are linearly dependent. This is in keeping with the fact that the subspace of generalized eigenvectors is of dimension 2, see (2.8). Also, one checks that for any phase $\beta \in]-\arccos \left(r^{2}-t^{2}\right), 0[\cup] 0, \arccos \left(r^{2}-t^{2}\right)[$,

$$
\left.\alpha^{-1}(\beta)=\left\{x_{1}, x_{2},-x_{2}-x_{1}\right\} \subset\right]-\pi, \pi[\text {. }
$$

Therefore, due to (6.12), only half these points contribute for the computation of the density of states. We can now compute the integrated density of states $N_{0}(\beta)$ as follows: Taking into account the normalisation by a factor $1 / 2 \pi$ in the definition (3.1), the fact that supp $k \subset\left[-\arccos \left(r^{2}-t^{2}\right), \arccos \left(r^{2}-t^{2}\right)\right]$ and the symmetries, we have for any $\beta \in\left[-\arccos \left(r^{2}-t^{2}\right), 0\right]$

$$
\begin{aligned}
N_{0}(\beta) & =\frac{1}{4 \pi} \int_{\mathbb{T}} d \lambda \chi_{\{-\alpha(\lambda)<\beta \leq 0\}}=\frac{1}{2 \pi} \int_{-\pi / 2}^{\pi / 2} d \lambda \chi_{\left\{\cos (2 \lambda)>\left(r^{2}-\cos (\beta)\right) / t^{2}\right\}} \\
& =\frac{1}{2 \pi} \int_{0}^{\arccos \left(\left(r^{2}-\cos (\beta)\right) / t^{2}\right)}=\frac{1}{2 \pi} \arccos \left(\frac{r^{2}-\cos (\beta)}{t^{2}}\right) .
\end{aligned}
$$

A similar computation for $\beta \in\left[0, \arccos \left(r^{2}-t^{2}\right)\right)$ yields (4.18). Therefore, $d k_{0}$ is absolutely continuous w.r.t. Lebesgue and, for any $|\lambda|<\arccos \left(r^{2}-t^{2}\right), d k_{0}(\lambda)=N^{\prime}(\lambda) d \lambda$, from which the result on the density of states follows. In order to obtain the Lyapunov exponent, it is enough to observe that the transfer matrices (2.8) $T$, now independent of $k$, are of determinant one and trace equal to $2\left(r^{2}-\cos (\lambda)\right) / t^{2}$. Therefore, it is readily checked that when the eigenvalues $\tau_{ \pm}(\lambda)$ of $T$

$$
\tau_{ \pm}(\lambda)=\left(r^{2}-\cos (\lambda) \pm \sqrt{\left(r^{2}-\cos (\lambda)\right)^{2}-t^{4}}\right) / t^{2}
$$

are complex conjugates, i.e. when $|\lambda|<\arccos \left(r^{2}-t^{2}\right)$, they are of modulus one, whereas

$$
\max \left\{\left|\tau_{+}\right|,\left|\tau_{-}\right|\right\}=\left(r^{2}-\cos (\lambda) \pm \sqrt{\left(r^{2}-\cos (\lambda)\right)^{2}-t^{4}}\right) / t^{2}
$$

if $|\lambda| \geq \arccos \left(r^{2}-t^{2}\right)$. It remains to use definition (2.13) to get $\gamma_{0}\left(e^{i \lambda}\right)$. In order to prove the last statement, we first rewrite the right hand side of Thouless formula with $d k_{0}\left(\lambda^{\prime}\right)$ above as

$$
\frac{1}{2 \pi} \int_{-1}^{1} \frac{\ln \left((x-y)^{2}\right)}{\sqrt{1-x^{2}}} d x+\ln 2
$$

by means elementary manipulations, changing variables to $x=\left(r^{2}-\cos \left(\lambda^{\prime}\right)\right) / t^{2}$ and introducing $y=\left(r^{2}-\cos (\lambda)\right) / t^{2} \in\left[-1,\left(r^{2}+1\right) / t^{2}\right]$. Hence we are to show that (6.18) above equals 0 if $y \leq 1$ and $\ln \left(y+\sqrt{y^{2}-1}\right)$ if $y>1$. We first deal with the case $y>1$. We can differentiate (6.18) with respect to $y$ under the integral sign to get

$$
\frac{1}{\pi} \int_{-1}^{1} \frac{d x}{\sqrt{1-x^{2}}(y-x)}=\frac{1}{2 \pi} \int_{C} \frac{d z}{\sqrt{1-z^{2}}(y-z)},
$$

where $C$ is a contour in the complex plane surrounding the segment $[-1,1]$ in the positive direction which does not contain $y$ in its interior. By deforming the contour to a circle centered at the origin and of radius $R>0$ large enough, we pick a residue at $y$. As the integral on the circle is of order $1 / R$, we eventually get in the limit $R \rightarrow \infty$

$$
\frac{d}{d y}\left\{\frac{1}{2 \pi} \int_{-1}^{1} \frac{\ln \left((x-y)^{2}\right)}{\sqrt{1-x^{2}}} d x+\ln 2\right\}=\frac{1}{\sqrt{y^{2}-1}}
$$


as expected. The limit as $y \rightarrow 1^{+}$of the Lyapunov exponent fixes the constant to 0 . Now, if $y \in]-1,1$ [, we first convert (6.18) to a contour integral along a path similar to the one above with te following difference. As the $\ln$ is multivalued, with a cut from $y$ along the real axis towards $-\infty$, the contour is attached to the point -1 . By assumption, $y$ does not belong to the contour of integration, so that we can now differentiate with respect to $y$ under the integral sign and thus get the same contour integral (6.19) as above to consider. However, by expanding the contour to infinity, we get to residue this time, so that (6.18) is constant for $y \in]-1,1[$. As it is known (GR], \# 4.224, p.526) that,

$$
\frac{1}{2 \pi} \int_{-1}^{1} \frac{\ln \left(x^{2}\right)}{\sqrt{1-x^{2}}} d x=\frac{2}{\pi} \int_{0}^{1} \frac{\ln (x)}{\sqrt{1-x^{2}}} d x=\frac{2}{\pi} \int_{0}^{\pi / 2} \ln (\sin (t)) d t=-\ln 2,
$$

we have, by continuity, that the integral is equal to zero on $[-1,1]$.

Proof of Lemma 4.2 ,

We use freely the notations above. Let us introduce the eigenprojectors $P_{ \pm}(x)$ associated with $\lambda_{ \pm}(x)$ such that

$$
V(x)=P_{+}(x) \lambda_{+}(x)+P_{-}(x) \lambda_{-}(x) .
$$

These quantities are analytic in $x$, in a strip including the real axis. Let $f \in C\left(S^{1}\right)$ and let us compute by means of (6.9) and the definition of $L_{ \pm}^{2}(\mathbb{T})$

$$
\begin{aligned}
& \operatorname{tr}\left\langle\chi_{M, N} \mid f\left(U_{0}\right) \chi_{M, N}\right\rangle=\sum_{M<j \leq N}\left\langle\varphi_{j} \mid f\left(U_{0}\right) \varphi_{j}\right\rangle= \\
& \sum_{\substack{j \text { even } \\
M<j \leq N}} \frac{1}{2 \pi} \int_{\mathbb{T}}\left\langle\left(\begin{array}{l}
1 \\
0
\end{array}\right) \mid\left(f\left(\lambda_{+}(x)\right) P_{+}(x)+f\left(\lambda_{-}(x)\right) P_{-}(x)\right)\left(\begin{array}{l}
1 \\
0
\end{array}\right)\right\rangle d x+ \\
& \quad \sum_{\substack{j \text { odd } \\
M<j \leq N}} \frac{1}{2 \pi} \int_{\mathbb{T}}\left\langle\left(\begin{array}{l}
0 \\
1
\end{array}\right) \mid\left(f\left(\lambda_{+}(x)\right) P_{+}(x)+f\left(\lambda_{-}(x)\right) P_{-}(x)\right)\left(\begin{array}{l}
0 \\
1
\end{array}\right)\right\rangle d x .
\end{aligned}
$$

The summand being independent of $j$ and uniformly bounded, we can rewrite the above trace as $N-M$ gets large as

$$
\begin{aligned}
& \frac{N-M}{4 \pi} \int_{\mathbb{T}} f\left(\lambda_{+}(x)\right) \operatorname{tr} P_{+}(x)+f\left(\lambda_{-}(x)\right) \operatorname{tr} P_{-}(x) d x+O(1)= \\
& \frac{N-M}{4 \pi} \int_{\mathbb{T}} f\left(\lambda_{+}(x)\right)+f\left(\lambda_{-}(x)\right) d x+O(1) .
\end{aligned}
$$

Hence, with $\lambda_{ \pm}(x)=e^{ \pm i \alpha(x)}$ as in (6.10), and taking into account the properties of $\alpha$, we get

$$
\begin{aligned}
\int_{\mathbb{T}} f\left(e^{i \lambda}\right) d k_{0}(\lambda) & =\frac{1}{4 \pi} \int_{\mathbb{T}} f\left(e^{i \alpha(x)}\right)+f\left(e^{-i \alpha(x)}\right) d x \\
& =\frac{1}{2 \pi} \int_{-\pi / 2}^{\pi / 2} f\left(e^{i \alpha(x)}\right)+f\left(e^{-i \alpha(x)}\right) d x
\end{aligned}
$$

which is easily seen to coincide with the "direct" definition of $d k_{0}$ in the above proof.

Proof of Proposition [5.2;

As in that case a commun term $\frac{1}{2^{n}}$ can be factorized, see (5.17), we compute the generating 
function of $\left|\mathcal{C}_{n-1}(j)\right|$, the cardinal of the set of relevant indices. Using the same symbols as above, we consider this time

$$
P_{n}(x)=\sum_{-2 n \leq j \leq 2 n}\left|\mathcal{C}_{n-1}(j)\right| x^{j}
$$

which we split into two parts $P_{n}(x)=P_{n}^{+}(x)+P_{n}^{-}(x)$ that satisfy for $n=0,1$,

$$
P_{0}^{+}(x)=1, P_{0}^{-}(x)=0, P_{1}^{+}(x)=1+x^{-2}, P_{1}^{-}(x)=x+x^{-1} .
$$

As above,

Lemma 6.1 For any $n \geq 0$,

$$
\left(\begin{array}{c}
P_{n+1}^{+}(x) \\
P_{n+1}^{-}(x)
\end{array}\right)=\left(\begin{array}{cc}
1+x^{-2} & x+x^{-1} \\
x+x^{-1} & 1+x^{2}
\end{array}\right)\left(\begin{array}{c}
P_{n}^{+}(x) \\
P_{n}^{-}(x)
\end{array}\right)
$$

with $P_{0}^{+}(x)=1, P_{0}^{-}(x)=0$.

By diagonalization of the corresponding transfer matrix, we get

$$
T^{n}(x)=A(x)\left(\begin{array}{cc}
0 & 0 \\
0 & \left(x^{-1}+x\right)^{2 n}
\end{array}\right) A(x)^{-1}
$$

where

$$
A(x)=\left(\begin{array}{cc}
1+x^{2} & x+x^{-1} \\
-\left(x+x^{-1}\right) & 1+x^{2}
\end{array}\right)
$$

and we compute

$$
\left(\begin{array}{c}
P_{n}^{+}(x) \\
P_{n}^{-}(x)
\end{array}\right)=T^{n}(x)\left(\begin{array}{l}
1 \\
0
\end{array}\right)=\frac{\left(x^{2}+1\right)^{2 n-1}}{x^{2 n}}\left(\begin{array}{l}
1 \\
x
\end{array}\right) .
$$

Using the binomial Theorem we obtain for $P_{n}^{ \pm}(x)$

$$
\begin{aligned}
& P_{n}^{+}(x)=\sum_{l=-n}^{n-1} x^{2 l}\left(\begin{array}{c}
2 n-1 \\
l+n
\end{array}\right) \\
& P_{n}^{-}(x)=\sum_{l=-n}^{n-1} x^{2 l+1}\left(\begin{array}{c}
2 n-1 \\
l+n
\end{array}\right),
\end{aligned}
$$

hence the end result.

\section{Acknowledgements:}

It is a pleasure to thank O.Bourget and R.Bacher for useful discussions and D.Damanik for pointing out reference [CMV] to me. 


\section{References}

[ADE] J. Asch , P. Duclos, P. Exner, Stability of driven systems with growing gaps, quantum rings, and Wannier ladders , J. Stat. Phys., 92 , 1053-1070 (1998).

[BB] G. Blatter, D. Browne, Zener tunneling and localization in small conducting rings, Phys. Rev. B, 37, (1988), 3856.

[BHJ] O. Bourget, J.S. Howland and A. Joye, Spectral Analysis of Unitary Band Matrices, Commun. Math. Phys., 234, (2003), 191-227.

[Be] J. Bellissard, Stability and Instability in quantum mechanics, in Trends and Developments in the Eighties, 1-106, Albeverio and Blanchard eds, World Scientific (1985).

[Bo] O. Bourget, Floquet Operators with Singular Continuous Spectrum, J. Math. Anal. Appl., 276, 28-39, (2002).

[CFKS] H.L. Cycon, R.G. Froese, W. Kirsch, B. Simon, Schrödinger Operators, Springer Verlag, 1987.

[CMV] M.J. Cantero, L. Moral and L. Velázquez, Five-Diagonal Matrices and Zeos of Orthogonal Polynomials on the Unit Circle, Linear Algebra and Its Applications, 326 C, 29-56, (2003)

[CL] R. Carmona, J. Lacroix, Spectral theory of random Schrodinger Operators, Birkhauser, 1990.

[Co1] M. Combescure, The Quantum Stability Problem for Time-periodic Perturbations of the Harmonic Oscillator, Ann. Inst. H. Poincaré, 47, 451-454, (1987).

[Co2] M. Combescure, Spectral properties of a periodically kicked quantum Hamiltonian, J. Stat. Phys., vol. 59, 679-690 (1990).

[Co3] M. Combescure, Recurrent versus diffusive quantum behaviour for time dependent Hamiltonians, Operator theory: advances and applications, vol. 57, Birkhauser Verlag (1992).

[CS] W. Craig; B. Simon, Subharmonicity of the Lyaponov Index, Duke Math.Jour. 50, 551-560, (1983).

[DF] S. DeBièvre, G. Forni, Transport properties of kicked and quasiperiodic Hamiltonians, J. Statist. Phys. 90, 1201-1223, (1998).

[DLSV] P. Duclos, O. Lev, P. Stovicek, M. Vittot, Weakly regular Hamiltonians with pure point spectrum, Rev. Math. Phys. 14, 531-568 (2002).

[DS] P. Duclos, P. Stovicek, Floquet Hamiltonians with pure point spectrum, Commun. Math. Phys., vol. 177, 327-347 (1996).

[GY] S. Graffi, K. Yajima, Absolute Continuity of the Floquet Spectrum for a nonlinearly Forced Harmonic Oscillator, Comm. Math. Phys., vol. 215, n 2, (2000) 245-250.

[GR] I.S.Gradshteyn, I.M.Ryzhik, Table of Integrals, Series, and Products, 4th Edition, Academic Press, 1965.

[GT] J.S.Geronimo, A.Teplyaev, A Difference Equation Arising from the Trigonometric Moment Problem Having Random Reflection Coefficients-An Operator Theoretic Approach, J. Func. Anal., 123, (1994), 12-45. 
[Ho1] J. Howland, Quantum Stability, in "Schrödinger Operators" Lecture Notes in Physics, E.Baslev Edt. Springer 403, 101-122, (1992).

[Ho2] J. Howland, Floquet operators with singular continuous spectrum, I, Ann. Inst. H. Poincaré Phys. Théor., vol. 49, 309-323 (1989).

[Ho3] J. Howland, Floquet operators with singular continuous spectrum, II, vol. 49, 325-334 (1989);

[Ho4] J. Howland, Floquet operators with singular continuous spectrum, III, vol. 69, 265-273 (1998).

[J] A. Joye, Absence of absolutely continuous spectrum of Floquet operators, J. Stat. Phys., vol. 75, 929-952 (1994).

[N1] G. Nenciu, Floquet operators without absolutely continuous spectrum, Ann. Inst. H. Poincaré Phys. Théor., vol. 59, 91-97 (1993).

[N2] G. Nenciu, Adiabatic theory: stability of systems with increasing gaps, Ann. Inst. H. Poincaré Phys. Théor., vol. 67, 411-424 (1997).

[Yaf] D.Yafaev, Mathematical Scattering Theory, General Theory, Transl. of Math.Mono. 105, 1992. 